\title{
INVOLUTIONS WITH ISOLATED FIXED POINTS ON ORIENTABLE 3-DIMENSIONAL FLAT SPACE FORMS
}

BY

\author{
E. LUFT AND D. SJERVE
}

\begin{abstract}
In this paper we completely classify (up to conjugacy) all involutions $\iota$ : $M \rightarrow M$, where $M$ is an orientable connected flat 3-dimensional space form, such that $\iota$ has fixed points but only finitely many. If $M_{1}, \ldots, M_{6}$ are the 6 space forms then only $M_{1}, M_{2}, M_{6}$ admit such involutions. Moreover, they are unique up to conjugacy. The main idea behind the proof is to find incompressible tori $T \subseteq M$ so that either $\iota(T)=T$ or $\iota(T) \cap T=\varnothing$ and then cut $M$ into simpler pieces. These results lead to a complete classification of 3-manifolds containing $\mathbf{Z} \oplus \mathbf{Z} \oplus \mathbf{Z}$ in their fundamental groups.
\end{abstract}

1. Introduction. In [1] it was shown that the only possible finitely generated abelian subgroups of the fundamental group of a 3-manifold are $\mathbf{Z}_{n}, \mathbf{Z} \oplus \mathbf{Z}_{2}, \mathbf{Z}$, $\mathbf{Z} \oplus \mathbf{Z}$, and $\mathbf{Z} \oplus \mathbf{Z} \oplus \mathbf{Z}$. Moreover, those manifolds $M^{3}$ such that $\pi_{1}(M)$ contains a finite subgroup or $\mathbf{Z} \oplus \mathbf{Z}_{2}$ as a subgroup were also characterized. In order to characterize those $M^{3}$ so that $\pi_{1}(M)$ contains $\mathbf{Z} \oplus \mathbf{Z} \oplus \mathbf{Z}$ as a subgroup it is necessary to determine the involutions with isolated fixed points on 3-dimensional orientable flat space forms (see [7] for this characterization).

The purpose of this paper is to classify up to conjugacy all involutions $\iota: M \rightarrow M$, where $M$ is an orientable flat 3-dimensional space form and $\iota$ has fixed points but only finitely many.

Throughout the paper we use the term space form as an abbreviation for a connected closed orientable 3-dimensional flat space form. According to Wolf (see [11, p. 117]) there are only 6 space forms up to affine equivalence. We use the notation $M_{1}, \ldots, M_{6}$ to correspond to the order of these space forms on p. 117 of [11]. In particular $M_{1}$ is just the torus $S^{1} \times S^{1} \times S^{1}$ and $M_{6}$ is the so-called Hantzsche-Wendt manifold [2]. It turns out that $M_{1}, \ldots, M_{5}$ are torus bundles over $S^{1}$, whereas $M_{6}$ is the union along the boundaries of twisted orientable $I$-bundles over a Klein bottle [8]. For a complete topological description see [7].

In $\$ 5$ we classify all involutions with isolated fixed points, on orientable torus bundles over $S^{1}$. The result is that only $M_{1}$ and $M_{2}$ admit such involutions and they are unique up to conjugacy. Then in $\S 6$ we carry out the same program for unions along the boundaries of two orientable twisted $I$-bundles over Klein bottles. Among these is also the space form $M_{2}$. We prove that besides $M_{2}$ only $M_{6}$ admits

Received by the editors November 17, 1982 and, in revised form, August 2, 1983.

1980 Mathematics Subject Classification. Primary 57S25. 
involutions with isolated fixed points. Again the involutions are uniquely determined up to conjugacy. These involutions on $M_{1}, M_{2}, M_{6}$ have 8,4,2 fixed points, respectively. For further descriptions see [7].

If $\iota: M_{i} \rightarrow M_{i}, i=1,2$, or 6 , is such an involution and $x_{1}, \ldots, x_{k}$ are the fixed points $(k=8,4,2)$ then there are disjoint 3-cells $C_{1}, \ldots, C_{k}$ so that $x_{i} \in \operatorname{int} C_{i}$ and $\iota\left(C_{i}\right)=C_{i}, 1 \leqslant i \leqslant k$. We let $M_{i}^{*}$ denote $M_{i}-\operatorname{int}\left(C_{1} \cup \cdots \cup C_{k}\right) / \iota$ the orbit manifold. In $\S 7$ we give explicit descriptions of $M_{1}^{*}, M_{2}^{*}$, and $M_{6}^{*}$.

All the space forms have incompressible tori and thus a certain hierarchy. The main idea behind the proofs is to find incompressible tori which either are disjoint from their image or which are invariant under the involution; thereby reducing the study of involutions on the various classes of manifolds to the study of involutions on simpler manifolds such as $T \times[0,1]$, where $T$ is the 2-dimensional torus, or $W=$ the twisted orientable $I$-bundle over the Klein bottle.

In $\S 3$, we determine all involutions with only isolated fixed points on $T \times[0,1]$ and then in $\$ 4$ we do this for $W$.

For the torus $M=S^{1} \times S^{1} \times S^{1}$ a complete description of all possible involutions was given in [4]. Another relevant reference is [10].

We would like to thank the referee for his comments.

2. Notation and preliminaries. We will work throughout in the PL category. Our references are [3 or 10]. A PL homeomorphism we simply call an isomorphism.

All involutions are assumed to have only isolated fixed points. The following lemma will be applied several times.

Lemma (2.1). Suppose $M$ is an n-manifold, $F \subseteq M$ is a proper bicollared $(n-1)$ submanifold, and $\iota: M \rightarrow M$ is an involution with $\iota(F)=F$. If $U$ is any neighborhood of $F$ in $M$, then there is a bicollar $F \times[-1,1]$ of $F=F \times 0$ in $M$ such that $F \times[-1,1] \subset U$ and $\iota(F \times[-1,1])=F \times[-1,1]$.

Moreover, we may assume that $F \times[-1,1] \cap \partial M=\partial F \times[-1,1]$.

Proof. There is a simplicial subdivision of $M$ containing $F$ as a subcomplex, with its simplicial neighborhood in $U$, and such that $\iota$ is simplicial. Let $F \times[-1,1]$ be a regular neighborhood with respect to this subdivision. Q.E.D.

We have the following

Proposition (2.2). Suppose $M$ is an n-manifold and $\iota: M \rightarrow M$ is an involution with only isolated fixed points. Then the fixed points of $\iota$ are in the interior of $M$.

Proof. There is a simplicial subdivision of $M$ containing the fixed points as vertices such that $\iota$ is simplicial. If a fixed point is on the boundary of $M$, its link is an $(n-1)$-cell and it is invariant under $\iota$. Therefore $\iota$ must have a fixed point on this link, and hence keep a 1-cell pointwise fixed; a contradiction. Q.E.D.

We will frequently apply the Lefschetz fixed point theorem as follows.

LEMMA (2.3). Let $M$ be a compact $n$-manifold and let $\iota: M \rightarrow M$ be an involution with fixed points $x_{1}, \ldots, x_{k}$. If $\lambda$ is the Lefschetz number of $\iota$, then $\lambda=k$. 
Proof. Choose a simplicial subdivision of $M$ containing $x_{1}, \ldots, x_{k}$ as vertices and such that $\iota$ is simplicial. The star-neighborhoods of $x_{1}, \ldots, x_{k}$ in the first barycentric subdivision then define disjoint 3-cells $C_{1}, \ldots, C_{k} \subset$ int $M$ with $x_{i} \in$ int $C_{i}$ and with $\iota\left(C_{i}\right)=C_{i}, 1 \leqslant i \leqslant k$. Since $\left.\iota\right|_{\partial C_{i}}, 1 \leqslant i \leqslant k$, is a fixed point free involution of the $(n-1)$-sphere $\partial C_{i},\left.\iota\right|_{\partial C_{i}}$ is orientation reversing if $n$ is odd, and orientation preserv-

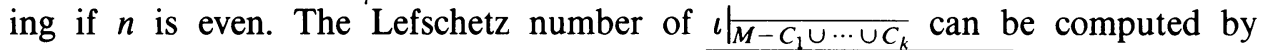
applying a Mayer-Vietoris sequence to $M=\frac{M-C_{1} \cup \cdots \cup C_{k}}{M-C_{1} \cup C_{k}} \cup\left(C_{1} \cup \cdots \cup\right.$

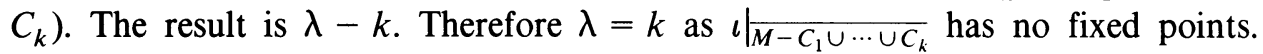
Q.E.D.

Note that if $k \geqslant 1$ and $M$ is orientable then $\iota$ must be orientation reversing if $n$ is odd and orientation preserving if $n$ is even.

Two involutions $\iota, \iota^{\prime}: X \rightarrow X$ on a space $X$ are said to be conjugate if there exists an isomorphism $h: X \rightarrow X$ with $\iota^{\prime}=h^{-1} \iota h$.

A 3-manifold $M$ is said to be irreducible if each 2-sphere in $M$ bounds a 3-cell in M.

Let $M$ be a 3-manifold and let $F \subset M$ be a compact 2-manifold which is either properly embedded in $M$ or is contained in $\partial M$. We say that $F$ is incompressible in $M$ if no component of $F$ is a 2-sphere and if for each 2-cell $B \subset M$ with $B \cap F=\partial B$ there is a 2-cell $B^{\prime} \subset F$ with $\partial B^{\prime}=\partial B$.

Definition. A proper annulus $A$ in a 3-manifold $M$ is said to be trivial if $A$ decomposes $M$ into a solid torus $V=D^{2} \times S^{1}$ and a submanifold $M_{0}$ such that:

(I) $M=M_{0} \cup V, M_{0} \cap V=\partial M_{0} \cap \partial V=A$, and

(II) there exists a nonseparating proper 2-cell $B \subseteq V$ so that $B \cap A=\partial B \cap A$ is a nonseparating proper 1-cell in $A$. Otherwise we say that $A$ is nontrivial.

Note that if the bounding 1-spheres of $A$ are in different boundary components of $M$ or if $A$ does not separate $M$ then $A$ is nontrivial.

A nontrivial, incompressible, proper annulus in a 3-manifold we call an essential annulus. (Compare remark in [6].)

Note a nonseparating proper annulus in an irreducible 3-manifold with incompressible boundary is essential.

We now summarize for the convenience of the reader the results of [6] which are the basic tools of our proofs.

Suppose $M$ is an irreducible 3-manifold and $\iota: M \rightarrow M$ is an involution. In Theorems (2.6) and (2.7) assume in addition that $M$ is orientable.

THEOREM (2.4). If there exists a proper nonseparating 2-cell $B_{0} \subset M$, then there exists one with $B \cap \iota(B)=\varnothing$.

Moreover, if $\partial B_{0} \cap \iota\left(\partial B_{0}\right)=\varnothing$ we may assume that $\partial B=\partial B_{0}$.

TheOrem (2.5). Suppose $A_{0} \subset M$ is an essential annulus with $\partial A_{0} \cap \iota\left(\partial A_{0}\right)=\varnothing$. Then either there is an essential annulus $A \subset M$ with

$$
A \cap \iota(A)=\varnothing \quad \text { and } \quad \partial A \cup \iota(\partial A)=\partial A_{0} \cup \iota\left(\partial A_{0}\right) \text {. }
$$

or there are two disjoint essential annuli $A_{1}, A_{2}$ in $M$ with $\iota\left(A_{i}\right)=A_{i}, i=1,2$, with $\partial\left(A_{1} \cup A_{2}\right)=\partial A_{0} \cup \iota\left(\partial A_{0}\right)$, and with no fixed points of $\iota$ on $A_{1} \cup A_{2}$. 
TheOrem (2.6). Suppose $A_{0} \subset M$ is an essential annulus. Let $\partial A_{0}=S_{01} \cup S_{02}$, and let $R_{i} \subset \partial M$ be the component with $S_{0 i} \subset R_{i}, i=1,2$. If $R_{1}=R_{2}$, assume that $R_{1}$ is incompressible. Then there is an essential annulus $A \subset M$ with either $A \cap \iota(A)=\varnothing$ or $\iota(A)=A$, and with $\partial A \cup \iota(\partial A) \subset R_{1} \cup R_{2} \cup \iota\left(R_{1} \cup R_{2}\right)$. In the latter case there are no fixed points of $\iota$ on $A$.

Furthermore, if $R_{1} \neq R_{2}$ and $\iota\left(R_{1} \cup R_{2}\right)=R_{1} \cup R_{2}$, we may assume that $\partial A=S_{1}$ $\cup S_{2}$ satisfies $S_{i} \subset R_{i}, i=1,2$.

THEOREM (2.7). Suppose $\iota: M \rightarrow M$ is an orientation reversing involution. If $M$ contains an incompressible torus, then $M$ contains an incompressible torus $T$ with either $T \cap \iota(T)=\varnothing$ or $\iota(T)=T$. In the latter case there are no fixed points of $\iota$ on $T$.

3. Involutions on $T \times[0,1]$. In order to describe the involutions on $T \times[0,1]$, where $T$ is the 2-dimensional torus $S^{1} \times S^{1}$, we introduce the following involutions.

Let $\alpha: S^{1} \rightarrow S^{1}$ be the antipodal map, $\alpha(x)=-x$; let $\kappa: S^{1} \rightarrow S^{1}$ denote conjugation, $\kappa(x)=\bar{x}$; and let $\tau:[0,1] \rightarrow[0,1]$ be the map $\tau(t)=1-t$. Note that $\alpha$ is fixed point free, $\kappa$ has 2 fixed points $\{1\}$ and $\{-1\}$, and $\tau$ has one fixed point $\left\{\frac{1}{2}\right\}$.

The main result of this section is in essence that any involution $\iota: T \times[0,1] \rightarrow T$ $\times[0,1]$ is conjugate to exactly one of the following 5 involutions of $T \times[0,1]$ :

$\alpha \times$ id $\times$ id, $\alpha \times \kappa \times$ id, $\alpha \times \kappa \times \tau, \quad \alpha \times$ id $\times \tau, \quad \kappa \times \kappa \times \tau$.

The first 4 involutions are fixed point free and the last one has exactly 4 fixed points. There is also an all important relative version. If $\iota$ has no fixed points this result also follows from [3, Theorem 10.3, p. 91].

We now give more detailed descriptions of the above 5 standard involutions on $T \times[0,1]$. In $T \times[0,1]$ we select annuli $A_{ \pm}$and solid tori $V_{ \pm}$as follows:

$$
\begin{aligned}
& A_{ \pm}=\{( \pm i, y, t) \in T \times[0,1]\}, \\
& V_{+}=\{(x, y, t) \in T \times[0,1] ; \operatorname{Re} x \geqslant 0\}, \\
& V_{-}=\{(x, y, t) \in T \times[0,1] ; \operatorname{Re} x \leqslant 0\} .
\end{aligned}
$$

Then $T \times[0,1]=V_{+} \cup V_{-}$and $V_{+} \cap V_{-}=A_{+} \cup A_{-}$(see Figure 1).

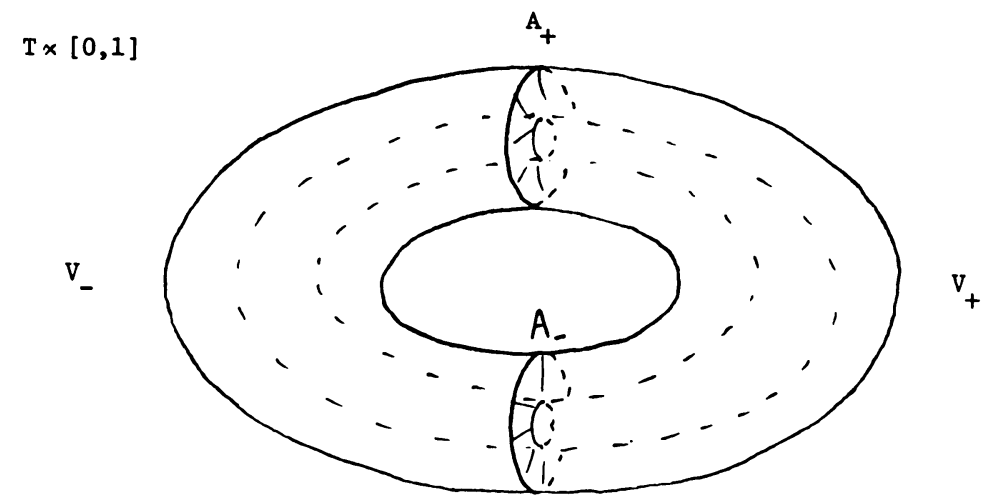

Figure 1 
Each of the 5 standard involutions maps $A_{+}$onto $A_{-}$. We have the following table:

\begin{tabular}{|c|c|c|c|c|}
\hline involution & orientation type & fixed points & image of $T \times 0$ & image of $V_{+}$ \\
\hline \hline$\alpha \times$ id $\times$ id & preserving & 0 & $T \times 0$ & $V_{-}$ \\
$\alpha \times \kappa \times$ id & reversing & 0 & $T \times 0$ & $V_{-}$ \\
$\alpha \times \kappa \times \tau$ & preserving & 0 & $T \times 1$ & $V_{-}$ \\
$\alpha \times$ id $\times \tau$ & reversing & 0 & $T \times 1$ & $V_{-}$ \\
$\kappa \times \kappa \times \tau$ & reversing & 4 & $T \times 1$ & $V_{+}$ \\
\hline
\end{tabular}

The first 3 columns of the table (i.e. the orientation type, the cardinality of the fixed point set, and the image of $T \times 0$ ) are conjugacy class invariants. Conversely, by our main result, if $\iota: T \times[0,1] \rightarrow T \times[0,1]$ is an involution, these 3 invariants determine uniquely its conjugacy class.

To classify the involutions of $T \times[0,1]$ we proceed as follows. First we classify the involutions on a solid torus. If $\iota: T \times[0,1] \rightarrow T \times[0,1]$ is an involution we show that there exists an incompressible annulus $A \subset T \times[0,1]$ that meets both boundary components of $T \times[0,1]$ and so that $A \cap \iota(A)=\varnothing$. Then $A \cup \iota(A)$ decomposes $T \times[0,1]$ into handlebodies $V_{ \pm}$as in the situation of the 5 standard involutions of $T \times[0,1]$. Applying the classification of the involutions on a solid torus will then lead to a classification of the involutions on $T \times[0,1]$.

In order to classify the involutions on a solid torus we begin with the following lemma, which is an immediate consequence of Theorem 1 in [5].

Lemma (3.1). Suppose ı, $\iota^{\prime}: S^{2} \times[0,1] \rightarrow S^{2} \times[0,1]$ are fixed point free involutions leaving the ends invariant. Then there exists an isomorphism $h: S^{2} \times[0,1] \rightarrow S^{2} \times$ $[0,1]$ so that

$$
\iota^{\prime}=h \iota h^{-1} \text {. }
$$

Furthermore, if we are given an isomorphism $h_{0}: S^{2} \times 0 \rightarrow S^{2} \times 0$ satisfying $\left.\iota^{\prime}\right|_{S^{2} \times 0}=h_{0} \iota h_{0}^{-1}$, then we may assume that $\left.h\right|_{S^{2} \times 0}=h_{0}$.

(Thus such an involution is conjugate to the involution $(x, t) \rightarrow(-x, t)$.)

TheOREM (3.2). Let $C$ be a 3-cell and let $\iota, \iota^{\prime}: C \rightarrow C$ be involutions. Then there exists an isomorphism $h: C \rightarrow C$ so that $\iota^{\prime}=h \iota^{-1}$.

Moreover, if $h_{0}: \partial C \rightarrow \partial C$ is an isomorphism satisfying $\left.\iota^{\prime}\right|_{\partial C}=h_{0} \iota h_{0}^{-1}$, we may assume that $\left.h\right|_{\partial C}=h_{0}$.

Proof. Note that since $\partial C / \iota \mid$ and $\partial C / \iota^{\prime} \mid$ are projective planes, there always exists an isomorphism $h_{0}: \partial C \rightarrow \partial C$ with $\left.\iota^{\prime}\right|_{\partial C}=h_{0} \iota h_{0}^{-1}$. Thus we may assume that $h_{0}$ is given.

By Lemma (2.3), $\iota, \iota^{\prime}$ have exactly 1 fixed point each (and they must be orientation reversing).

Let $K, K^{\prime}$ be simplicial subdivisions of $C$ so that $\iota: K \rightarrow K, \iota^{\prime}: K^{\prime} \rightarrow K^{\prime}$ are simplicial. Then the fixed points $x_{0}, x_{0}^{\prime}$ of $\iota, \iota^{\prime}$ are vertices of $K, K^{\prime}$, respectively. Let $K_{0}=\operatorname{St}\left(x_{0}, K\right), K_{0}^{\prime}=\operatorname{St}\left(x_{0}^{\prime}, K^{\prime}\right)$ be the star neighborhoods of $x_{0}, x_{0}^{\prime}$ in $K, K^{\prime}$, 
respectively. Then $\iota\left(K_{0}\right)=K_{0}, \iota^{\prime}\left(K_{0}^{\prime}\right)=K_{0}^{\prime}$ and $\left.\iota\right|_{K_{0}},\left.\iota^{\prime}\right|_{K_{0}^{\prime}}$ are conings of $\left.\iota\right|_{\partial K},\left.\iota^{\prime}\right|_{\partial K_{0}^{\prime}}$, respectively. We may assume that $K_{0} \cap \partial C=\varnothing=K_{0}^{\prime} \cap \partial C$. It then follows that $W=\overline{C-\left|K_{0}\right|}, W^{\prime}=\overline{C-\left|K_{0}^{\prime}\right|}$ are copies of $S^{2} \times[0,1]$.

By Lemma (3.1) we can extend $h_{0}$ to an isomorphism $\hat{h}_{0}: W \rightarrow W^{\prime}$ so that $\left.\iota^{\prime}\right|_{W^{\prime}}=\hat{h}_{0} \iota \hat{h}_{0}^{-1}$. By coning $\hat{h}_{0}|:| \partial K_{0}|\rightarrow| \partial K_{0}^{\prime} \mid$ to the vertices $x_{0}, x_{0}^{\prime}$, we extend $\hat{h}_{0}$ to an isomorphism $h: C \rightarrow C$. Then $\iota^{\prime}=h \iota h^{-1}$. Q.E.D.

Thus there is a unique conjugacy class of involutions on the 3-cell $C$. For example, let $C=c\left(S^{2}\right)$ ( = the cone on $\left.S^{2}\right)$ and let $\alpha: S^{2} \rightarrow S^{2}$ be the antipodal map. Then $\hat{\alpha}$ : $C \rightarrow C, \hat{\alpha}(x, t)=(\alpha(x), t)$, is an involution with one fixed point. If $C=D^{2} \times[0,1]$, then a canonical involution is $(x, t) \rightarrow(-x, 1-t)$.

Now we are ready to classify the involutions on the solid torus $V=D^{2} \times S^{1}$.

THEOREM (3.3). Suppose $\iota, \iota^{\prime}: V \rightarrow V$ are involutions and one of the following holds:

(I) $\iota, \iota$ are orientation preserving,

(II) $\iota, \iota^{\prime}$ are orientation reversing and fixed point free,

(III) $\iota, \iota^{\prime}$ are orientation reversing and have fixed points.

Then there is an isomorphism $h: V \rightarrow V$ so that $\iota^{\prime}=h \iota^{-1}$.

Assume $h_{0}: \partial V \rightarrow \partial V$ is an isomorphism with $\left.\iota^{\prime}\right|_{\partial V}=h_{0} \iota h_{0}^{-1}$. In addition, for case (I) only, assume that $h_{0}$ extends to an isomorphism of $V$ onto $V$. Then $h: V \rightarrow V$ can be chosen to satisfy $h \mid \partial V=h_{0}$.

Proof. Since both $\partial V / \iota \mid$ and $\partial V / \iota^{\prime} \mid$ are either tori or Klein bottles there always exists an isomorphism $h_{0}: \partial V \rightarrow \partial V$ so that $\left.\iota^{\prime}\right|_{\partial V}=h_{0} \iota h_{0}^{-1}$ and if $\iota, \iota^{\prime}$ are orientation preserving, extending to an isomorphism of $V$ onto $V$. Thus we assume that $h_{0}$ : $\partial V \rightarrow \partial V$ is given.

(1) First we show that if $\iota, \iota^{\prime}$ are orientation reversing, $h_{0}$ extends to an isomorphism of $V$ onto $V$.

We have $H_{1}(\partial V)=\mathbf{Z}[\partial D] \oplus \mathbf{Z}[S]$.

Lemma (2.3) implies immediately that $\iota$ has a fixed point if and only if $\iota_{*}$ : $H_{1}(V) \rightarrow H_{1}(V)$ is $\iota_{*}=-$ id (in which case it has exactly two fixed points). Therefore $(\iota \mid)_{*},\left(\iota^{\prime} \mid\right)_{*}: H_{1}(\partial V) \rightarrow H_{1}(\partial V)$ have the following descriptions:

If $\iota, \iota^{\prime}$ are fixed point free, then

$$
\begin{array}{cl}
(\iota \mid)_{*}[\partial D]=-[\partial D], & \left(\iota^{\prime} \mid\right)_{*}[\partial D]=-[\partial D], \\
(\iota \mid)_{*}[S]=\alpha[\partial D]+[S], & \left(\iota^{\prime} \mid\right)_{*}[S]=\alpha^{\prime}[\partial D]+[S]
\end{array}
$$

and if $\iota, \iota^{\prime}$ have fixed points, then

$$
\begin{array}{cl}
(\iota \mid)_{*}[\partial D]=[\partial D], & \left(\iota^{\prime} \mid\right)_{*}[\partial D]=[\partial D], \\
(\iota \mid)_{*}[S]=\beta[\partial D]-[S], & \left(\iota^{\prime} \mid\right)_{*}[S]=\beta^{\prime}[\partial D]-[S] .
\end{array}
$$

Let $h_{0 *}[\partial D]=a[\partial D]+b[S], h_{0 *}[S]=c[\partial D]+d[S]$ with $a \cdot d-b \cdot c= \pm 1$. Then we must have

$$
\left[\begin{array}{cc}
-1 & 0 \\
\alpha^{\prime} & 1
\end{array}\right]\left[\begin{array}{ll}
a & b \\
c & d
\end{array}\right]=\left[\begin{array}{ll}
a & b \\
c & d
\end{array}\right]\left[\begin{array}{cc}
-1 & 0 \\
\alpha & 1
\end{array}\right]
$$


in the first case, and

in the second case.

$$
\left[\begin{array}{ll}
1 & 0 \\
\beta^{\prime} & -1
\end{array}\right]\left[\begin{array}{ll}
a & b \\
c & d
\end{array}\right]=\left[\begin{array}{ll}
a & b \\
c & d
\end{array}\right]\left[\begin{array}{cc}
1 & 0 \\
\beta & -1
\end{array}\right]
$$

In both cases we obtain $-b=b$ and hence $b=0$. Therefore $h_{0 *}[\partial D]= \pm[\partial D]$. Consequently $h_{0}$ extends.

(2) By Theorem (2.4) there is a nonseparating proper 2-cell $B \subset V$ with

$$
B \cap \iota(B)=\varnothing \text {. }
$$

Then $B \cup \iota(B)$ decomposes $V$ into 3-cells $C_{+}, C_{-}: V=C_{+} \cup C_{-}, C_{+} \cap C_{-}=\partial C_{+} \cap$ $\partial C_{-}=B \cup \iota(B)$. (See Figure 2).

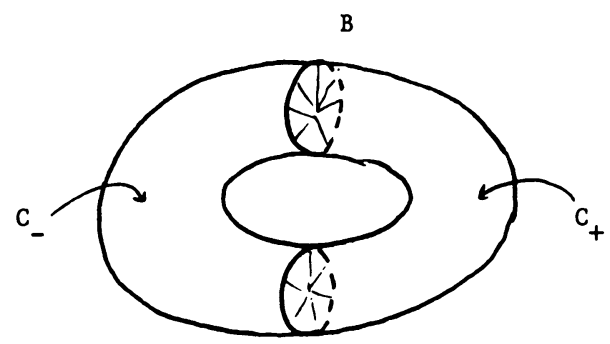

l(B)

FigURE 2

We have $\iota^{\prime} h_{0}(\partial B)=h_{0} \iota(\partial B)$ and hence

$$
h_{0}(\partial B) \cap \iota^{\prime}\left(h_{0}(\partial B)\right)=\varnothing .
$$

By (1), and in case (I) by the hypothesis on $h_{0}$, there is a proper 2-cell $B^{\prime} \subset V$ with $\partial B^{\prime}=h_{0}(\partial B)$. By Theorem (2.4) we may assume that $B^{\prime} \cap \iota^{\prime}\left(B^{\prime}\right)=\varnothing$.

Then $B^{\prime} \cup \iota^{\prime}\left(B^{\prime}\right)$ decomposes $V$ again into 3-cells $C_{+}^{\prime}, C_{-}^{\prime}: V=C_{+}^{\prime} \cup C_{-}^{\prime}, C_{+}^{\prime} \cap C_{-}^{\prime}$ $=\partial C_{+}^{\prime} \cap \partial C_{-}^{\prime}=B^{\prime} \cup \iota^{\prime}\left(B^{\prime}\right)$.

Suppose that $h_{0}\left(C_{+} \cap \partial V\right)=C_{+}^{\prime} \cap \partial V$. Let $h_{01}: B \rightarrow B^{\prime}$ be an extension of $h_{0} \mid$ : $\partial B \rightarrow \partial B^{\prime}$ and define $h_{02}: \iota(B) \rightarrow \iota^{\prime}\left(B^{\prime}\right)$ by equivariance $h_{02}=\left.\iota^{\prime} h_{0} \iota\right|_{\iota(B)}$. Define $\hat{h}_{0}=h_{01} \cup h_{02}: B \cup \iota(B) \rightarrow B^{\prime} \cup \iota^{\prime}\left(B^{\prime}\right)$. By construction, $\left.\iota^{\prime}\right|_{B^{\prime} \cup \iota^{\prime}\left(B^{\prime}\right)}=\hat{h}_{0} \iota \hat{h}_{0}^{-1}$.

Case 1. Both $\iota$ and $\iota^{\prime}$ have no fixed points.

As observed in (1), then $\iota_{*}, \iota_{*}^{\prime}: H_{1}(V) \rightarrow H_{1}(V)$ are $\iota_{*}=\iota_{*}^{\prime}=$ id. Consequently, $\iota\left(C_{+}\right)=C_{-}$and $\iota^{\prime}\left(C_{+}^{\prime}\right)=C_{-}^{\prime}$.

Let $h_{+}: C_{+} \rightarrow C_{+}^{\prime}$ be an extension of $\left.h_{0}\right|_{C_{+} \cap \partial V} \cup \hat{h}_{0}: \partial C_{+} \rightarrow \partial C_{+}^{\prime}$ and define $h_{-}$: $C_{-} \rightarrow C_{-}^{\prime}$ by $h_{-}=\iota^{\prime} h_{+} \iota_{C_{-}}$. Then $h_{+}$and $h_{-}$agree on $C_{+} \cap C_{-}=B \cup \iota(B)$ and therefore piece together to give the conjugating isomorphism $h=h_{+} \cup h_{-}: V \rightarrow V$ with $\iota^{\prime}=h \iota h^{-1}$.

Case 2. Both $\iota$ and $\iota^{\prime}$ have fixed points.

Then necessarily $\iota\left(C_{i}\right)=C_{i}$ and $\iota^{\prime}\left(C_{i}^{\prime}\right)=C_{i}^{\prime}, i=+,-$. By Theorem (3.2) the isomorphism $\left.h_{0}\right|_{C_{i} \cap \partial V} \cup \hat{h}_{0}: \partial C_{i} \rightarrow \partial C_{i}^{\prime}$ extends to an isomorphism $h_{i}: C_{i} \rightarrow C_{i}^{\prime}$ with 
$\left.\iota^{\prime}\right|_{C_{i}^{\prime}}=h_{i} \iota h_{i}^{-1}, i=+,-$. Again $h_{+}$and $h_{-}$agree on $C_{+} \cap C_{-}=B \cup \iota(B)$ and hence $h=h_{+} \cup h_{-}: V \rightarrow V$ is an isomorphism with $\iota^{\prime}=h \iota h^{-1}$. Q.E.D.

Here are explicit representatives for the 3 conjugacy classes of involutions on $V$. Recall that $\alpha: S^{1} \rightarrow S^{1}$ is the antipodal map and $\kappa: S^{1} \rightarrow S^{1}$ is conjugation. Let $\hat{\alpha}, \hat{\kappa}$ : $D^{2} \rightarrow D^{2}$ denote the radial extensions. These are again involutions. The involution $\hat{\alpha}$ has precisely one fixed point. Then we have the following 3 involutions of $V=D^{2}$ $\times S^{1}$ :

$$
\begin{array}{ll}
\omega=\hat{\alpha} \times \alpha, & \omega(x, y)=(-x,-y), \\
\iota_{0}=\hat{\kappa} \times \alpha, & \iota_{0}(x, y)=(\bar{x},-y), \\
\iota_{2}=\hat{\alpha} \times \kappa, & \iota_{2}(x, y)=(-x, \bar{y}) .
\end{array}
$$

We select proper 2-cells $B_{ \pm}$and 3-cells $C_{ \pm}$as follows:

$$
\begin{aligned}
& B_{ \pm}=\{(x, \pm i) \in D \times S\}, \\
& C_{+}=\{(x, y) \in D \times S ; \operatorname{Re} y \geqslant 0\}, \\
& C_{-}=\{(x, y) \in D \times S ; \operatorname{Re} y \leqslant 0\} .
\end{aligned}
$$

Then $V=C_{+} \cup C_{-}$and $C_{+} \cap C_{-}=B_{+} \cup B_{-}$. Compare Figure 2. Each of the 3 standard involutions maps $B_{+}$onto $B_{-}$. We have the following table:

\begin{tabular}{|c|c|c|c|}
\hline involution & $\begin{array}{c}\text { orientation } \\
\text { type }\end{array}$ & fixed points & image of $C_{+}$ \\
\hline \hline$\omega=\hat{\alpha} \times \alpha$ & preserving & 0 & $C_{-}$ \\
$\iota_{0}=\hat{\kappa} \times \alpha$ & reversing & 0 & $C_{-}$ \\
$\iota_{0}=\hat{\alpha} \times \kappa$ & reversing & 2 & $C_{+}$ \\
\hline
\end{tabular}

The first 2 columns of the table (i.e. the orientation type, the cardinality of the fixed point set) are conjugacy class invariants. Conversely, by Theorem (3.3), if $\iota$ : $V \rightarrow V$ is an involution, these 2 invariants determine uniquely its conjugacy class.

COROllary (3.4). An involution $\iota: V \rightarrow V$ is conjugate to:

(I) $\omega$, if $\iota$ is orientation preserving,

(II) $\iota_{0}$, if $\iota$ is orientation reversing and fixed point free, and

(III) $\iota_{2}$, if $\iota$ is orientation reversing with fixed points.

Also there is a relative version as in (3.3).

REMARK. If $\iota: V \rightarrow V$ is a fixed point free involution then $V / \iota$ is a solid torus again if $\iota$ is orientation preserving and a solid Klein bottle if $\iota$ is orientation reversing.

(Of course this result also follows immediately from Dehn's Lemma. It can also be used to prove cases (I) and (II) of Theorem (3.3).)

Next we continue with some technical lemmas. Recall that $T$ denotes the 2-dimensional torus $S^{1} \times S^{1}$.

LEMMA (3.5). Let $A \subset T \times[0,1]$ be a proper annulus. Then the following three statements are equivalent. 
(I) $A$ is essential.

(II) $A$ is incompressible and meets both boundary components of $T \times[0,1]$.

(III) $A$ is nonseparating.

Lemma (3.6). (1) Suppose $A \subset T \times[0,1]$ is an essential annulus. Then $(T \times[0,1], A)$ is isomorphic to $\left(S^{1} \times S^{1} \times[0,1], x_{0} \times S^{1} \times[0,1]\right)$.

(2) Suppose $A_{1}, A_{2} \subset T \times[0,1]$ are disjoint essential annuli. Then $(T \times$ $\left.[0,1], A_{1}, A_{2}\right)$ is isomorphic to $\left(S^{1} \times S^{1} \times[0,1], x_{1} \times S^{1} \times[0,1], x_{2} \times S^{1} \times[0,1]\right)$.

Proof. (1) We may assume that $x_{0} \times S^{1} \times 0$ is a boundary component of $A$ and that $S^{1} \times y_{0} \times[0,1]$ and $A$ intersect transversally in a single proper 1-cell in $A$ and in $S^{1} \times y_{0} \times[0,1]$. With this it is not difficult to construct an isomorphism

$$
(T \times[0,1], A) \rightarrow\left(S^{1} \times S^{1} \times[0,1], x_{0} \times S^{1} \times[0,1]\right) .
$$

(2) By (1) we may assume that

$$
(T \times[0,1], A)=\left(S^{1} \times S^{1} \times[0,1], x_{1} \times S^{1} \times[0,1]\right) .
$$

We may assume that $S^{1} \times y_{0} \times[0,1]$ and $A_{2}$ intersect transversally in a single proper 1-cell in $A_{2}$ and in $S^{1} \times y_{0} \times[0,1]$. Then it is not difficult to construct an isomorphism

$$
\left(T \times[0,1], A_{1}, A_{2}\right) \rightarrow\left(S^{1} \times S^{1} \times[0,1], x_{1} \times S^{1} \times[0,1], x_{2} \times S^{1} \times[0,1]\right) .
$$

Q.E.D.

Main Lemma (3.7). Suppose $\iota: T \times[0,1] \rightarrow T \times[0,1]$ is an involution. Then there exists an essential annulus $A \subset T \times[0,1]$ with $A \cap \iota(A)=\varnothing$.

Proof. By Theorem (2.6) there is an essential annulus $A_{0} \subset T \times[0,1]$ with either $A_{0} \cap \iota\left(A_{0}\right)=\varnothing$ or $\iota\left(A_{0}\right)=A_{0}$. In the latter case there are no fixed points of $\iota$ on $A_{0}$. If $A_{0} \cap \imath\left(A_{0}\right)=\varnothing$, let $A=A_{0}$. Hence suppose $\iota\left(A_{0}\right)=A_{0}$.

By Lemma (2.1) there is a bicollar $A_{0} \times[-\varepsilon, \varepsilon]$ of $A_{0}=A_{0} \times 0$ in $T \times[0,1]$ with $\left(A_{0} \times[-\varepsilon, \varepsilon]\right) \cap \partial(T \times[0,1])=\partial A_{0} \times[-\varepsilon, \varepsilon]$ and $\iota\left(A_{0} \times[-\varepsilon, \varepsilon]\right)=A_{0} \times[-\varepsilon, \varepsilon]$. If $\iota\left(A_{0} \times \varepsilon\right)=A_{0} \times-\varepsilon$, let $A=A_{0} \times \varepsilon$. Hence suppose $\iota\left(A_{0} \times \varepsilon\right)=A_{0} \times \varepsilon$ and thus $\iota\left(A_{0} \times-\varepsilon\right)=A_{0} \times-\varepsilon$. According to Lemma (3.6) we may assume that

$$
\left(T \times[0,1], A_{0}\right)=\left(S^{1} \times S^{1} \times[0,1], x_{0} \times S^{1} \times[0,1]\right) .
$$

Let $I \subset A_{0}$ be a nonseparating proper 1-cell with $I \cap \iota(I)=\varnothing$. (Consider $A_{0} / \iota$. It is either an annulus or a Moebius strip. A nonseparating proper 1-cell in $A_{0} / \iota \mid$ lifts to a nonseparating proper 1-cell $I$ as required.) By choosing $\varepsilon$ sufficiently small we can insure that $\iota$ has no fixed points on $A_{0} \times[-\varepsilon, \varepsilon]$ and

$$
(I \times[-\varepsilon, \varepsilon]) \cap \iota(I \times[-\varepsilon, \varepsilon])=\varnothing .
$$

Now consider the solid torus $V=\overline{T \times[0,1]-A_{0} \times[-\varepsilon, \varepsilon]}$. We have $\iota(V)=V$. Suppose $B \subset V$ is a proper 2-cell with

$$
B \cap\left(A_{0} \times[-\varepsilon, \varepsilon]\right)=I \times \varepsilon \cup I \times-\varepsilon \text { and } B \cap \iota(B)=\varnothing .
$$

Then $A=I \times[-\varepsilon, \varepsilon] \cup B$ is an essential annulus with $A \cap \iota(A)=\varnothing$.

We now show the existence of such a 2-cell $B$. 
Note that the inclusions $A_{0} \times i \varepsilon \rightarrow V, i=1,-1$, induce isomorphisms

$$
H_{1}\left(A_{0} \times i \varepsilon\right) \rightarrow H_{1}(V), \quad i=1,-1 .
$$

It follows from Lemma $(2.3)$ that $(\iota \mid)_{*}: H_{1}\left(A_{0} \times i \varepsilon\right) \rightarrow H_{1}\left(A_{0} \times i \varepsilon\right)$ is $(\iota \mid)_{*}=$ id. By commutativity then $\left(\left.\iota\right|_{V}\right)_{*}: H_{1}(V) \rightarrow H_{1}(V)$ is $\left(\left.\iota\right|_{V}\right)_{*}=$ id. By Corollary (3.5), $V / \iota \mid$ is a solid torus or a solid Klein bottle. Let $p: V \rightarrow V / \iota \mid$ be the covering projection, and let $\bar{B} \subset V / l \mid$ be a nonseparating proper 2-cell. We may assume that $\bar{B} \cap$ $p\left(A_{0} \times i \varepsilon\right)=p(I \times i \varepsilon), i=1,-1$. Let $B \subset p^{-1}(\bar{B})$ be the 2-cell with $B \cap A_{0} \times \varepsilon=$ $I \times \varepsilon$. If $B \cap A_{0} \times-\varepsilon=I \times-\varepsilon, B$ has the required property. If

$$
B \cap A_{0} \times-\varepsilon=\iota(I \times-\varepsilon),
$$

we replace $\bar{B}$ by a nonseparating proper 2-cell $B^{\prime}$ so that $B^{\prime} \cap p\left(A_{0} \times i \varepsilon\right)=$ $p(I \times i \varepsilon), i=1,-1, B^{\prime} \cap \bar{B}=p(I \times \varepsilon) \cup p(I \times-\varepsilon)$, and $H_{1}\left(B^{\prime} \cup \bar{B}\right) \rightarrow H_{1}(V / \iota)$ is an isomorphism. Again let $B \subset p^{-1}\left(B^{\prime}\right)$ be the 2-cell with $B \cap A_{0} \times \varepsilon=I \times \varepsilon$. This time $B \cap A_{0} \times-\varepsilon=I \times-\varepsilon$, and $B$ has the required property. Q.E.D.

Now suppose $\iota: T \times[0,1] \rightarrow T \times[0,1]$ is an involution. By the preceding Main Lemma there exists an essential annulus $A \subset T \times[0,1]$ satisfying $A \cap \iota(A)=\varnothing$.

By Lemma (3.6), (2), we may assume that

$$
(T \times[0,1], A, \iota(A))=\left(S_{1} \times S_{2} \times[0,1], x_{+} \times S_{2} \times[0,1], x_{-} \times S_{2} \times[0,1]\right) .
$$

Let $S_{1}=I_{+} \cup I_{-}, I_{+}, I_{-} 1$-cells with $I_{+} \cap I_{-}=\partial I_{+} \cap \partial I_{-}=\left\{x_{+}, x_{-}\right\}$, and let $V_{i}=$ $I_{i} \times S_{2} \times[0,1], i=+,-$. The solid tori $V_{+}, V_{-}$decompose $T \times[0,1]:$

$$
T \times[0,1]=V_{+} \cup V_{-} \text {and } V_{+} \cap V_{-}=\partial V_{+} \cap \partial V_{-}=A \cup \iota(A) .
$$

Compare Figure 1.

We have $H_{1}(T \times[0,1])=\mathbf{Z}\left[S_{1}\right] \oplus \mathbf{Z}\left[S_{2}\right]$.

LEMMA (3.8). If $\iota: T \times[0,1] \rightarrow T \times[0,1]$ is fixed point free, then we may assume that $\iota\left(V_{+}\right)=V_{-}$.

Proof. Suppose $\iota\left(V_{+}\right)=V_{+}$and hence $\iota\left(V_{-}\right)=V_{-}$. Let $p: T \times[0,1] \rightarrow T \times[0,1] / \iota$ be the covering projection. Then $p\left(V_{+}\right), p\left(V_{-}\right)$are solid tori or solid Klein bottles. Choose a nonseparating proper 1-cell $I \subset A$. Let $\bar{B}_{i} \subset p\left(V_{i}\right)$ be a nonseparating proper 2-cell with $\bar{B}_{i} \cap p(A)$ consisting of exactly 2 proper 1-cells, one of them being $p(I)$, the other we denote by $\bar{I}, i=+,-$. Let $B_{i} \subset p^{-1}\left(\bar{B}_{i}\right)$ be the 2-cell with $B_{i} \cap A=I, i=+,-$. Then $B_{i} \cap \iota(A) \subset p^{-1}(\bar{I}), i=+,-$.

We may assume that $B_{+} \cap \imath(A)=B_{-} \cap \imath(A)$. Otherwise we replace $\bar{B}_{-}$by a nonseparating proper 2 -cell $\bar{B}_{-}^{\prime}$ satisfying $\bar{B}_{-}^{\prime} \cap p(A)=\bar{B}_{-} \cap p(A)=\bar{B}_{-} \cap \bar{B}_{-}^{\prime}$ and $H_{1}\left(\bar{B}_{-} \cup \bar{B}_{-}^{\prime}\right) \rightarrow H_{1}\left(p\left(V_{-}\right)\right)$is an isomorphism.

The proper annulus $A^{\prime}=B_{+} \cup B_{-}$is incompressible, meets both boundary components of $T \times[0,1]$, and satisfies $A^{\prime} \cap \iota\left(A^{\prime}\right)=\varnothing$ and $A^{\prime} \cap A=I$.

Now $A^{\prime} \cup \iota\left(A^{\prime}\right)$ decomposes $T \times[0,1]$ again into solid tori $V_{+}^{\prime}, V_{-}^{\prime}$ :

$$
T \times[0,1]=V_{+}^{\prime} \cup V_{-}^{\prime}, \quad V_{+}^{\prime} \cap V_{-}^{\prime}=\partial V_{+}^{\prime} \cap \partial V_{-}^{\prime}=A^{\prime} \cup \iota\left(A^{\prime}\right) .
$$

We claim that $\iota\left(V_{+}^{\prime}\right)=V_{-}^{\prime}$. Namely, we have the presentation $T \times 0=(T \times 0) \cap$ $A \times(T \times 0) \cap A^{\prime}$. If $\iota\left(V_{+}^{\prime}\right)=V_{+}^{\prime}$, then $\iota\left(V_{-}\right)=V_{-}^{\prime}$, and we conclude that

$$
\iota_{*}: H_{1}(T \times[0,1]) \rightarrow H_{1}(T \times[0,1])
$$


is $\iota_{*}=-\mathrm{id}$. The Lefschetz number of $\iota$ is $\neq 0$ and hence $\iota$ has fixed points. Contradiction. Q.E.D.

Lemma (3.9). If $\iota: T \times[0,1] \rightarrow T \times[0,1]$ has fixed points, then $\iota$ has exactly 4 fixed points, $\iota_{*}: H_{1}(T \times[0,1]) \rightarrow H_{1}(T \times[0,1])$ is $\iota_{*}=-\mathrm{id}, \iota(T \times 0)=T \times 1$.

Proof. Since $\iota$ has fixed points we must have $\iota\left(V_{i}\right)=V_{i}, i=+,-$.

Suppose a fixed point of $\iota$ is in $V_{+}$. Then, by Lemma (2.3), ( $\left.\iota\right)_{*}: H_{1}\left(V_{+}\right) \rightarrow H_{1}\left(V_{+}\right)$ must be $(\iota \mid)_{*}=-$ id. We conclude that $\iota_{*}\left[S_{2}\right]=-\left[S_{2}\right]$ and by commutativity that $(\iota \mid)_{*}: H_{1}\left(V_{-}\right) \rightarrow H_{1}\left(V_{-}\right)$is also $(\iota \mid)_{*}=-$ id. Therefore, by Lemma $(2.3), \iota$ has exactly 4 fixed points and its Lefschetz number is 4 . Hence $\iota_{*}\left[S_{1}\right]=-\left[S_{1}\right]+m\left[S_{2}\right]$. Since $\iota_{*}^{2}=$ id we conclude that $m=0$. Thus $\iota_{*}=-\mathrm{id}$. Since $\iota$ is orientation reversing we must have $\iota(T \times 0)=T \times 1$. Q.E.D.

TheOREM (3.10). Suppose $\iota, \iota^{\prime}: T \times[0,1] \rightarrow T \times[0,1]$ are involutions and one of the following holds:

(I) $\iota, \iota^{\prime}$ are orientation preserving and $\iota(T \times 0)=\iota^{\prime}(T \times 0)=T \times 0$,

(II) $\iota, \iota^{\prime}$ are orientation reversing and $\iota(T \times 0)=\iota^{\prime}(T \times 0)=T \times 0$,

(III) $\iota, \iota^{\prime}$ are orientation preserving and $\iota(T \times 0)=\iota^{\prime}(T \times 0)=T \times 1$,

(IV) $\iota, \iota^{\prime}$ are orientation reversing, $\iota(T \times 0)=\iota^{\prime}(T \times 0)=T \times 1$, and $\iota, \iota^{\prime}$ are fixed point free, or

(V) $\iota, \iota^{\prime}$ are orientation reversing, $\iota(T \times 0)=\iota^{\prime}(T \times 0)=T \times 1$, and $\iota, \iota^{\prime}$ have fixed points.

Then there is an isomorphism $h: T \times[0,1] \rightarrow T \times[0,1]$ so that $\iota^{\prime}=h \iota h^{-1}$.

Moreover, in case $(\mathrm{V})$, if $h_{0}: \partial(T \times[0,1]) \rightarrow \partial(T \times[0,1])$ is an isomorphism with

$$
\left.\iota^{\prime}\right|_{\partial(T \times[0,1])}=h_{0} \iota h_{0}^{-1}
$$

we may assume that $\left.h\right|_{\partial(T \times[0,1])}=h_{0}$.

(Note that if $h^{\prime}: T \times 0 \rightarrow T \times 0$ is an arbitrary isomorphism, then $h_{0}=h^{\prime} \cup$ $\left(\left.\iota^{\prime} h^{\prime} \iota\right|_{T \times 1}\right): \partial(T \times[0,1]) \rightarrow \partial(T \times[0,1])$ is the unique isomorphism that extends $h_{0}$ and satisfies $\left.\iota^{\prime}\right|_{\partial(T \times[0,1])}=h_{0} \iota h_{0}^{-1}$. Thus we may assume that $\left.h\right|_{T \times 0}=h^{\prime}$.)

Proof. (1) Suppose $\iota, \iota^{\prime}$ are fixed point free. Let $A, A^{\prime} \subset T \times[0,1]$ be essential annuli with $A \cap \iota(A)=A^{\prime} \cap \iota^{\prime}\left(A^{\prime}\right)=\varnothing$ and let $V_{ \pm}, V_{ \pm}^{\prime}$ be the corresponding solid tori. By Lemma (3.8) we may assume that $\iota\left(V_{+}\right)=V_{-}, \iota^{\prime}\left(V_{+}^{\prime}\right)=V_{-}^{\prime}$.

Choose an arbitrary isomorphism $h_{1}: A \rightarrow A^{\prime}$. Define $h_{2}: \iota(A) \rightarrow \iota^{\prime}(A)$ by equivariance, $h_{2}=\left.\iota^{\prime} h_{1} \iota\right|_{\iota(A)}$. It follows from the hypothesis in cases (I)-(IV) that $h_{1} \cup h_{2}: A \cup \iota(A) \rightarrow A^{\prime} \cup \iota^{\prime}\left(A^{\prime}\right)$ extends to an isomorphism $h_{+}: V_{+} \rightarrow V_{+}^{\prime}$. Define $h_{-}: V_{-} \rightarrow V_{-}^{\prime}$ by equivariance, $h_{-}=\left.\imath^{\prime} h_{+} \iota\right|_{V_{-}}$. Then $h_{+}$and $h_{-}$piece together to define an isomorphism $h=h_{+} \cup h_{-}: T \times[0,1] \rightarrow T \times[0,1]$. By construction, $\iota^{\prime}=h \iota h^{-1}$.

(2) Suppose $\iota, \iota^{\prime}$ have fixed points. By Lemma (3.5) this is case (V).

By the remark in the statement of the theorem we may assume that $h_{0}$ is given.

Let $A \subset T \times[0,1]$ be an essential annulus with $A \cap \iota(A)=\varnothing$. Let $V_{ \pm}$be the corresponding solid tori. Since $\iota$ has fixed points we must have $\iota\left(V_{i}\right)=V_{i}, i=+,-$.

There is a proper annulus $A_{0} \subset T \times[0,1]$ with $\partial A_{0}=h_{0} \partial A$. We have

$$
\partial A_{0} \cap \iota^{\prime}\left(\partial A_{0}\right)=h_{0}(\partial A) \cap h_{0} \iota(\partial A)=h_{0}(\partial A \cap \iota(\partial A))=\varnothing .
$$


By Theorem (2.5), there is either an essential annulus $A^{\prime} \subset T \times[0,1]$ with $\partial A^{\prime} \cup$ $\partial \iota^{\prime}\left(A^{\prime}\right)=\partial A_{0} \cup \partial \iota^{\prime}\left(A_{0}\right)$ and with $A^{\prime} \cap \iota^{\prime}\left(A^{\prime}\right)=\varnothing$, or there are two disjoint essential annuli $A_{i} \subset T \times[0,1]$ with $\iota^{\prime}\left(A_{i}\right)=A_{i}, i=1,2 ; \partial\left(A_{1} \cup A_{2}\right)=\partial A_{0} \cup \partial \iota^{\prime}\left(A_{0}\right)$; and with no fixed points of $\iota^{\prime}$ on $A_{1} \cup A_{2}$.

The second possibility cannot occur. Namely, if $\iota^{\prime}\left(A_{i}\right)=A_{i}$ the Lefschetz number of $\iota^{\prime} \mid: A_{i} \rightarrow A_{i}$ would have to be 2 by Lemma (3.9), and hence there would have to be fixed points of $\iota^{\prime}$ on $A_{i}$.

We may assume that $A^{\prime} \cap T \times 0=h_{0}(A \cap T \times 0)$. Then necessarily $A^{\prime} \cap T \times 1$ $=h_{0}(A \cap T \times 1)$. Thus $\partial A^{\prime}=h_{0}(\partial A)$.

Let $V_{ \pm}^{\prime}$ be the solid tori determined by $A^{\prime} \cup \iota^{\prime}\left(A^{\prime}\right)$. We may assume that $h_{0}\left(V_{i} \cap \partial(T \times[0,1])\right)=V_{i}^{\prime} \cap \partial(T \times[0,1]), i=+,-$. Since $\iota^{\prime}$ has fixed points we again must have $\iota^{\prime}\left(V_{i}^{\prime}\right)=V_{i}^{\prime}, i=+,-$.

The isomorphism $h_{0} \mid: \partial A \rightarrow \partial A^{\prime}$ can be extended to an isomorphism $h_{1}: A \rightarrow A^{\prime}$. The isomorphism $h_{2}: \iota(A) \rightarrow \iota^{\prime}(A)$ is defined by equivariance, $h_{2}=\iota^{\prime} h_{1} \iota l_{\iota(A)}$. Piecing together we obtain the isomorphisms

$$
h_{i}=\left.h_{0}\right|_{V_{i} \cap \partial(T \times[0,1])} \cup h_{1} \cup h_{2}: \partial V_{i} \rightarrow \partial V_{i}, \quad i=+,-,
$$

which by construction satisfy $\left.\iota^{\prime}\right|_{\partial V_{i}^{\prime}}=h_{i} \iota h_{i}^{-1}, i=+,-$.

By Theorem (3.3), $h_{i}$ extends to an isomorphism $\hat{h}_{i}: V_{i} \rightarrow V_{i}^{\prime}$ with $\left.\iota^{\prime}\right|_{V_{i}^{\prime}}=\hat{h}_{i} \iota \hat{h}_{i}^{-1}$, $i=+,-$.

Then $h=\hat{h}_{+} \cup \hat{h}_{-}: T \times[0,1] \rightarrow T \times[0,1]$ is the desired extension of $h_{0}$ with $\iota^{\prime}=h \iota h^{-1}$. Q.E.D.

There are also relative versions of the theorem in cases (I)-(IV). They follow easily from Corollary (3.12). Since they are not needed in this work they are omitted.

Corollary (3.11). Suppose $\iota: T \times[0,1] \rightarrow T \times[0,1]$ is an involution. Then $\iota$ is conjugate to:

(I) $\alpha \times$ id $\times$ id, if $\iota$ is orientation preserving and $\iota(T \times 0)=T \times 0$,

(II) $\alpha \times \kappa \times$ id, if $\iota$ is orientation reversing and $\iota(T \times 0)=T \times 0$,

(III) $\alpha \times \kappa \times \tau$, if $\iota$ is orientation preserving and $\iota(T \times 0)=T \times 1$.

(IV) $\alpha \times$ id $\times \tau$, if $\iota$ is orientation reversing, $\iota(T \times 0)=T \times 1$, and $\iota$ has no fixed points, or

(V) $\kappa \times \kappa \times \tau$, if $\iota$ is orientation reversing, $\iota(T \times 0)=T \times 1$, and $\iota$ has fixed points.

Moreover, in case $(\mathrm{V})$, if $h_{0}: \partial(T \times[0,1]) \rightarrow \partial(T \times[0,1])$ is an isomorphism with $\iota_{\partial(T \times[0,1])}=h_{0}(\kappa \times \kappa \times \tau) h_{0}^{-1}$, then $h_{0}$ extends to an isomorphism $h: T \times[0,1] \rightarrow T$ $\times[0,1]$ with $\iota=h(\kappa \times \kappa \times \tau) h^{-1}$.

Corollary (3.12). Suppose $\iota: T \times[0,1] \rightarrow T \times[0,1]$ is an involution without fixed points.

(I) If $\iota$ is orientation preserving and $\iota(T \times 0)=T \times 0$, then $T \times[0,1] / \iota=T \times$ $[0,1]$.

(II) If $\iota$ is orientation reversing and $\iota(T \times 0)=T \times 0$, then $T \times[0,1] / \iota=K \times$ [0,1], K a Klein bottle.

(III) If $\iota$ is orientation preserving and $\iota(T \times 0)=T \times 1$, then $T \times[0,1] / \iota=$ orientable twisted I-bundle over a Klein bottle.

(IV) If $\iota$ is orientation reversing and $\iota(T \times 0)=T \times 1$, then $T \times[0,1] / \iota=M O \times$ $S^{1}$, i.1O a Moebius strip. 
4. Involutions on the orientable twisted $I$-bundle over a Klein bottle. As a model for the oriented twisted $I$-bundle over a Klein bottle we take the following manifold $W$ :

$$
W=S^{1} \times[0,1] \times[-1,1] /(x, 0, t) \sim(\bar{x}, 1,-t) .
$$

Let $q: S^{1} \times[0,1] \times[-1,1] \rightarrow W$ be the natural map. Then $K=q\left(S^{1} \times[0,1] \times 0\right)$ is a Klein bottle and $\pi: W \rightarrow K, \pi(q(x, s, t))=q(x, s, 0)$ defines an $I$-bundle structure over the Klein bottle $K$.

$\partial W$ is a torus and $\pi \mid: \partial W \rightarrow K$ is the orientable double covering.

We introduce the annulus $A_{*}=q\left(S^{1} \times 0 \times[-1,1]\right)=q\left(S^{1} \times 1 \times[-1,1]\right)$. Then $A_{*}$ is nonseparating. By Corollary (4.3), this property determines $A_{*}$ uniquely up to an ambient isotopy.

The main result of this section is in essence that any involution $\iota: W \rightarrow W$ is conjugate to exactly one of the 3 involutions $\nu, \rho_{0}, \rho_{2}: W \rightarrow W$ :

$$
\begin{gathered}
\nu(q(x, s, t))=q(-x, s, t), \\
\rho_{0}(q(x, s, t))=q(-x, s,-t), \\
\rho_{2}(q(x, s, t))= \begin{cases}q\left(-\bar{x}, \frac{1}{2}-s,-t\right), & \text { if } 0 \leqslant s \leqslant \frac{1}{2}, \\
q\left(-x, \frac{3}{2}-s, t\right), & \text { if } \frac{1}{2} \leqslant s \leqslant 1 .\end{cases}
\end{gathered}
$$

There is also a relative version which is crucial for our application.

We now give more detailed descriptions of the above 3 standard involutions.

To describe $\rho_{2}$, we introduce the nonseparating, nontrivial, and incompressible annulus $A_{c}=q\left(S^{1} \times \frac{1}{2} \times[-1,1]\right)$. Then $A_{*} \cup A_{c}$ decomposes $W$ into 2 solid tori $V_{ \pm}:$

(See Figure 3.)

$$
W=V_{+} \cup V_{-} \text {and } V_{+} \cap V_{-}=A_{*} \cup A_{c} .
$$

Each of the 3 standard involutions maps $V_{i}$ onto itself, $i=+,-$.

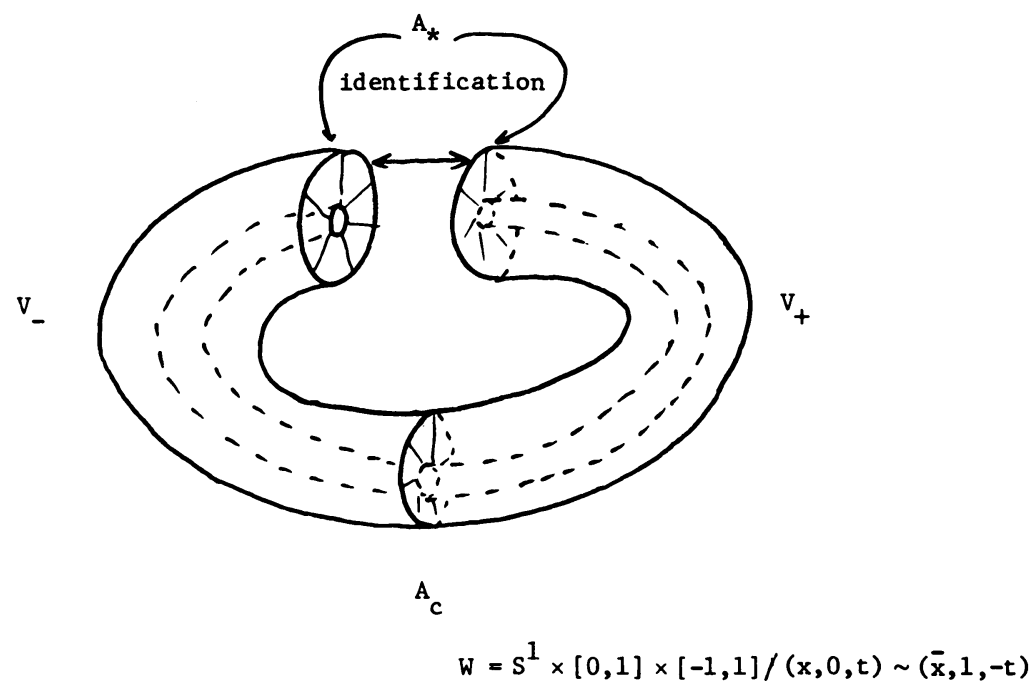

Figure 3 
We have the following table:

\begin{tabular}{|c|c|c|c|}
\hline involution & $\begin{array}{c}\text { orientation } \\
\text { type }\end{array}$ & fixed points & image of $A_{*}$ \\
\hline \hline$\nu$ & preserving & 0 & $A_{*}$ \\
$\rho_{0}$ & reversing & 0 & $A_{*}$ \\
$\rho_{2}$ & reversing & 2 & $A_{*}$ \\
\hline
\end{tabular}

The first 2 columns of the table (i.e. the orientation type and the cardinality of the fixed point set) are conjugation class invariants. Conversely, by the announced result, if $\iota: W \rightarrow W$ is an involution, these 2 invariants determine uniquely its conjugacy class.

If $\iota: W \rightarrow W$ is an involution, we will first find an annulus that has the same properties as $A_{*}$ does with regard to the 3 standard involutions. This is the essential step in the classification of the involutions of $W$.

LEMMA (4.1). Let $K$ be a Klein bottle and let $S_{1}, S_{2} \subset K$ be 2-sided nonseparating 1-spheres. Then there is an ambient isotopy on $K$ that maps $S_{1}$ onto $S_{2}$, the isotopy being a composition of ambient isotopies each the identity outside a 2-cell.

Proof. Let $S_{i}^{\prime} \subset K$ be a 1-sphere that intersects $S_{i}$ transversally in exactly 1 point. Then $S_{i}^{\prime}$ cannot be 2-sided. It follows then from [3, Lemma 2.13, p. 23], that there are embeddings $f_{i}: S^{1} \rightarrow K$ with $f_{i}\left(S^{1}\right)=S_{i}$ and with $f_{1}$ and $f_{2}$ homotopic.

Let $p: T \rightarrow K$ be the orientable double covering and let $\tilde{f}_{i}: S^{1} \rightarrow T$ be a lift of $f_{i}$, $i=1,2$, with $\tilde{f}_{1}$ and $\tilde{f}_{2}$ homotopic. We may assume that $\tilde{f}_{1}\left(S^{1}\right)$ and $\tilde{f}_{2}\left(S^{1}\right)$ intersect transversally with intersection number 0 . We can find a 2-cell $B$ with $\partial B \subset \tilde{f}_{1}\left(S^{1}\right) \cup$ $\tilde{f}_{2}\left(S^{1}\right)$ and with exactly 2 intersection points of $\tilde{f}_{1}\left(S^{1}\right)$ and $\tilde{f}_{2}\left(S^{1}\right)$ on $\partial B$. Then $p(B) \subset K$ is a 2-cell with $\partial p(B) \subset S_{1} \cup S_{2}$ and with exactly 2 intersection points of $S_{1}$ and $S_{2}$ on $\partial p(B)$. The 2-cell $p(B)$ can be used to eliminate these 2 intersection points.

Therefore we may assume that $S_{1} \cap S_{2}=\varnothing$. Then there is an annulus $A \subset K$ with $\partial A=S_{1} \cup S_{2}$ and the proof can be completed. Q.E.D.

Proposition (4.2). Suppose $A \subset W$ is an essential annulus. Then there is an ambient isotopy on $W$ mapping $A$ to $A_{1}$ so that $A_{1}$ and $K$ intersect transversally in $a$ single 2-sided 1-sphere $S^{1}$ with $\pi^{-1}\left(S^{1}\right)=A_{1}$.

If $A$ does not separate $W$, then we may assume that $A_{1}=A_{*}$.

Proof. We may assume that $A$ and $A_{*}$ intersect transversally. Then $A \cap A_{*}$ consists of disjoint proper 1-cells and 1-spheres.

If a 1-sphere of $A \cap A_{*}$ bounds a 2-cell in $A_{*}$ it must also bound a 2-cell in $A$ and vice versa. Since $W$ is irreducible these 1 -spheres can be removed by applying an ambient isotopy to $A$.

If there is a proper 1-cell of $A \cap A_{*}$ in $A_{*}$ that meets only one component of $\partial A_{*}$, then there is a 2-cell $D \subset A_{*}$ with $I=D \cap A=\partial D \cap A$ a proper 1-cell in $A$ and with $D \cap \partial W=\overline{\partial D-I}$. Since $A$ is nontrivial, it follows that there is a 2-cell $D^{\prime} \subset A$ 
with $D^{\prime} \cap D=\partial D^{\prime} \cap \partial D=I$ and with $D^{\prime} \cap \partial W=\overline{\partial D^{\prime}-I}$. There is a 2-cell $D^{\prime \prime} \subset \partial W$ with $\partial D^{\prime \prime}=\partial\left(D \cup D^{\prime}\right)$. Using the 3-cell bounded by the 2-sphere $D \cup D^{\prime}$ $\cup D^{\prime \prime}$ an ambient isotopy on $W$ can be constructed which applied to $A$ removes $I$ from $A \cap A_{*}$.

Thus we may assume that $A \cap A_{*}$ consists entirely of disjoint 1-spheres in $A, A_{*}$ that do not bound 2-cells in $A, A_{*}$, or disjoint proper 1-cells in $A, A_{*}$ that meet both boundary components of $A, A_{*}$, or $A \cap A_{*}=\varnothing$.

Suppose that $A \cap A_{*} \neq \varnothing$ consists of disjoint 1-spheres that do not bound 2-cells in $A, A_{*}$. Then there is an annulus $A^{\prime} \subset A$ with $\partial A^{\prime}=S_{1} \cup S_{2}, S_{2} \subset \partial A$, and $A^{\prime} \cap A_{*}=S_{1}$; and there is an annulus $A^{\prime \prime} \subset A_{*}$ with one component of $\partial A^{\prime \prime}$ being $S_{1}$, the other component being in $\partial W$, and such that annuli $A^{\prime}, A^{\prime \prime}$ determine a solid torus $V$ with $V \cap \partial W=\overline{\partial V-\left(A^{\prime} \cup A^{\prime \prime}\right)}$. This solid torus can be used to construct an ambient isotopy on $W$ which when applied to $A$ removes $S_{1}$ from $A \cap A_{*}$.

Now suppose that $A \cap A_{*} \neq \varnothing$ consists of disjoint proper 1-cells in $A, A_{*}$ that meet both boundary components of $A, A_{*}$. We have $A_{*}=q\left(S^{1} \times 0 \times[-1,1]\right)=$ $q\left(S^{1} \times 1 \times[-1,1]\right)$. Applying an ambient isotopy on $A_{*}$ to $A \cap A_{*}$, extending it to an ambient isotopy on $W$, and applying it to $A$, we may assume that

$$
A \cap A_{*}=q\left(x_{1} \times 0 \times[-1,1] \cup \cdots \cup x_{m} \times 0 \times[-1,1]\right) .
$$

The disjoint 1-cells of $A \cap A_{*}$ in $A$ decompose $A$ into 2-cells $B_{1}, \ldots, B_{m}$ with

$$
\begin{aligned}
B_{i} \cap A_{*}=\partial B_{i} \cap A_{*}=q\left(x_{i} \times 0 \times[-1,1]\right) \cup q\left(x_{i+1} \times 0 \times[-1,1]\right), & \\
i & =1, \ldots, m\left(\text { where } x_{m+1}=x_{i}\right) .
\end{aligned}
$$

Considering the proper 2-cells $\overline{q^{-1}\left(\text { int } B_{i}\right)} \subset S^{1} \times[0,1] \times[-1,1]$, we can find an ambient isotopy on $W$ keeping $A_{*}$ pointwise fixed and mapping $A$ to $A_{1}$ such that $A_{1} \cap K=S^{1}$ is a single 1 -sphere and such that $A_{1}=\pi^{-1}\left(S^{1}\right)$. Necessarily, $S^{1}$ is 2-sided.

If $A \cap A_{*}=\varnothing$, then there is an ambient isotopy on $W$ that maps $A$ to $A_{*}$.

Lastly, suppose that $A$ does not separate $W$. Then $S^{1}$ does not separate $K$. By Lemma (4.1), there is an ambient isotopy on $K$ that maps $S^{1}$ to $A_{*} \cap K$; the isotopy being a composition of ambient isotopies each the identity outside a 2-cell. Therefore this isotopy extends to an ambient isotopy of $W$ that maps $K$ onto itself, preserves the fibers of $\pi$, and hence maps $A_{1}$ to $A_{*}$. Q.E.D.

COROLlaRY (4.3). If $A \subset W$ is a nonseparating proper annulus, then there is an ambient isotopy on $W$ that maps $A$ onto $A_{*}$.

PROof. A nonseparating proper annulus is necessarily nontrivial and incompressible.

MaIn Lemma (4.4) Suppose $\iota: W \rightarrow W$ is an involution. Then there is a nonseparating proper annulus $A \subset W$ with either $A \cap \iota(A)=\varnothing$ or $\iota(A)=A$. In the latter case there are no fixed points of $\iota$ on $A$. If $A \cap \iota(A)=\varnothing$, then $\iota$ has exactly 2 fixed points. Conversely, if $\iota$ has a fixed point there is a nonseparating proper annulus $A \subset W$ with $A \cap \iota(A)=\varnothing$. 
Proof. By Theorem (2.6) there is an essential annulus $A_{0} \subset W$ with either $A_{0} \cap \iota\left(A_{0}\right)=\varnothing$ or $\iota\left(A_{0}\right)=A_{0}$ and in the latter case with no fixed points of $\iota$ on $A_{0}$.

(1) Suppose $A_{0}$ separates $W$ and $\iota\left(A_{0}\right)=A_{0}$. We show that there is a nonseparating proper annulus $A \subset W$ with $\iota(A)=A$ and with no fixed points of $\iota$ on $A$.

By Proposition (4.2) we may assume that $A_{0}$ and $K$ intersect transversally in a single 2-sided 1-sphere $S^{1}$ with $\pi^{-1}\left(S^{1}\right)=A_{0}$. Suppose $W=V_{+} \cup V_{-}$and $V_{+} \cap V_{-}=$ $\partial V_{+} \cap \partial V_{-}=A_{0}$. Then $V_{i} \cap K$ is a Moebius strip, $i=+,-$. We have $V_{i}=$ $\pi^{-1}\left(V_{i} \cap K\right)$. Therefore $V_{i}$ is a solid torus.

Case 1. $\iota\left(V_{+}\right)=V_{+}$. Since there are no fixed points of $\iota$ on $A_{0}$, we must have $(\iota \mid)_{*}$ : $H_{1}\left(A_{0}\right) \rightarrow H_{1}\left(A_{0}\right)$ is the identity.

Let $j: A_{0} \rightarrow V_{+}$be the inclusion. Then $j_{*} H_{1}\left(A_{0}\right)=2 \mathbf{Z} \subset H_{1}\left(V_{+}\right)=\mathbf{Z}$. Therefore, by commutativity, $(\iota)_{*}: H_{1}\left(V_{+}\right) \rightarrow H_{1}\left(V_{+}\right)$is also the identity. By Lemma (2.3), ८): $V_{+} \rightarrow V_{+}$has no fixed points. By Corollary (3.5) $V_{+} / \iota \mid$ is a solid torus or a solid Klein bottle.

Consider the inclusion $\bar{j}: A_{0} / \iota\left|\rightarrow V_{+} / \imath\right|$ and $\bar{j}_{*}: H_{1}\left(A_{0} / \iota \mid\right) \rightarrow H_{1}\left(V_{+} / \imath \mid\right)$. By commutativity $\bar{j}_{*} H_{1}\left(A_{0} / \iota\right)=2 \mathrm{Z} \subset H_{1}\left(V_{+} / \iota \mid\right)=\mathbf{Z}$. Therefore the inclusion $\bar{j}: A_{0} / \iota \mid$ $\rightarrow V_{+} / \iota$ lifts to two embeddings of $A_{0} / \iota$ into $\partial V_{+}$. Hence $A_{0} \cap \iota A_{0}=\varnothing$. A contradiction. Thus $\iota\left(V_{+}\right)=V_{+}$is not possible.

Case 2. $\iota\left(V_{+}\right)=V_{-}$. Consider the annulus or Moebius strip $A_{0} / \iota$ (depending on whether $\iota: A_{0} \rightarrow A_{0}$ is orientation preserving or orientation reversing, respectively). A nonseparating proper 1-cell in $A_{0} / \imath \mid$ determines a nonseparating proper 1-cell $I \subset A_{0}$ with $I \cap \iota(I)=\varnothing$. There is a nonseparating proper 2-cell $B \subset V_{+}$so that $B \cap A_{0}=I \cup \iota(I)$.

Define $A=B \cup \iota(B)$. Then $A$ is a nonseparating proper annulus with $\iota(A)=A$ and with no fixed points of $\iota$ on $A$.

(2) Suppose $A_{0}$ separates $W$ and $A_{0} \cap \iota\left(A_{0}\right)=\varnothing$.

We show again that there is a nonseparating proper annulus $A \subset W$ with $\iota(A)=A$ and with no fixed points of $\iota$ on $A$.

Again by Proposition (4.2), we may assume that $A_{0}$ and $K$ intersect transversally in a single 2-sided 1-sphere $S^{1}$ in $K$ with $\pi^{-1}\left(S^{1}\right)=A_{0}$.

Suppose that $W=V \cup V^{\prime}$ with $V \cap V^{\prime}=\partial V \cap \partial V^{\prime}=A_{0}$ and with $\iota\left(A_{0}\right) \subset V^{\prime}$. Since $V \cap K$ and $V^{\prime} \cap K$ are Moebius strips it follows that $V$ and $V^{\prime}$ are solid tori. Now $\iota\left(A_{0}\right)$ decomposes $V^{\prime}$ into two components, one being $\iota(V)$. Thus we have the following decomposition of $W$ into 3 solid tori $V_{0}, V, \iota(V)$ :

$$
W=V \cup V_{0} \cup \iota(V), \quad V \cap V_{0}=A_{0}, \quad \iota(V) \cap V_{0}=\iota\left(A_{0}\right), \quad V \cap \iota(V)=\varnothing .
$$

Therefore $\iota\left(V_{0}\right)=V_{0}$.

There is a nonseparating proper 2-cell $B_{0} \subset V_{0}$ such that $B_{0} \cap A_{0}$ is a nonseparating proper 1-cell in $A_{0}, B_{0} \cap \iota\left(A_{0}\right)$ is a nonseparating proper 1-cell in $\iota\left(A_{0}\right)$, and $B_{0} \cap \iota\left(B_{0}\right)=\varnothing$.

Proof. First suppose that $\iota \mid: V_{0} \rightarrow V_{0}$ has no fixed points. By Corollary (3.5) $V_{0} / \iota$ is a solid torus or a solid Klein bottle. Let $\bar{B}_{0} \subset V_{0} / \iota \mid$ be a nonseparating proper 2-cell with $\bar{B}_{0} \cap A_{0} \iota \mid$ consisting of 2 disjoint nonseparating proper 1-cells. Let $B_{0}$ be 
a lift of $\bar{B}_{0}$. Next suppose that $\iota: V_{0} \rightarrow V_{0}$ has fixed points. Define the involution $\iota_{2}$ : $S^{1} \times[0,1] \times[-1,1] \rightarrow S^{1} \times[0,1] \times[-1,1]$ by $\iota_{2}(x, s, t)=(\bar{x}, 1-s,-t)$. There is an isomorphism

$$
\begin{gathered}
h_{0}:\left(\partial\left(S^{1} \times[0,1] \times[-1,1]\right), S^{1} \times 0 \times[-1,1], S^{1} \times 1 \times[-1,1]\right) \\
\rightarrow\left(\partial V_{0}, A_{0}, \iota\left(A_{0}\right)\right)
\end{gathered}
$$

with $\left.\iota\right|_{\partial V_{0}}=h_{0} \iota_{2} h_{0}^{-1}$. By Theorem (3.3), $h_{0}$ extends to an isomorphism $h: S^{1} \times[0,1]$ $\times[-1,1] \rightarrow V_{0}$ with $\left.\iota\right|_{V_{0}}=h \iota_{2} h^{-1}$. Choose $s_{0} \in S^{1}$ with $\bar{s}_{0} \neq s_{0}$. Define

$$
B_{0}=h\left(s_{0} \times[0,1] \times[-1,1]\right) .
$$

There is a nonseparating proper 2-cell $B \subset V$ with $B \cap A_{0}=\left(B_{0} \cup \iota\left(B_{0}\right)\right) \cap A_{0}$.

Then $A=B \cup B_{0} \cup \iota\left(B \cup B_{0}\right)$ is a nonseparating proper annulus with $\iota(A)=A$ and with no fixed points of $\iota$ on $A$.

(3) Suppose that $A \subset W$ is a nonseparating proper annulus with $A \cap \iota(A)=\varnothing$. We show that $\iota$ has exactly 2 fixed points. For the following arguments we may assume that $A=A_{*}$.

$A \cup \iota(A)$ decomposes $W$ into 2 solid tori $V_{ \pm}$:

$$
W=V_{+} \cup V_{-} \text {and } V_{+} \cap V_{-}=\partial V_{+} \cap \partial V_{-}=A \cup \iota(A) \text {. }
$$

It cannot happen that $\iota\left(V_{+}\right)=V_{-}$. Namely if so, let $I \subset A$ be a nonseparating proper 1-cell and let $B \subset V_{+}$be a nonseparating proper 2-cell with $B \cap(A \cup \iota(A))$ $=I \cup \iota(I)$. Then $B \cup \iota(B)$ must be a Moebius strip. But $\iota \mid: B \cup \iota(B) \rightarrow B \cup \iota(B)$ is a fixed point free involution, a contradiction: A Moebius strip does not admit a fixed point free involution. (Otherwise, the quotient space would have to be a Moebius strip again. A 2-fold covering of a Moebius strip, however, is an annulus.)

Thus we must have $\iota\left(V_{+}\right)=V_{+}$and $\iota\left(V_{-}\right)=V_{-}$. We may assume that $V_{+}=$ $q\left(S^{1} \times\left[0, \frac{1}{2}\right] \times[-1,1]\right), V_{-}=q\left(S^{1} \times\left[\frac{1}{2}, 1\right] \times[-1,1]\right), \iota(A)=q\left(S^{1} \times \frac{1}{2} \times[-1,1]\right)$ (compare Figure 3). Consider the isomorphisms

$$
\begin{aligned}
& \iota_{+}=(q \mid)^{-1} \iota(q \mid): S^{1} \times\left[0, \frac{1}{2}\right] \times[-1,1] \rightarrow S^{1} \times\left[0, \frac{1}{2}\right] \times[-1,1], \\
& \iota_{-}=(q \mid)^{-1} \iota(q \mid): S^{1} \times\left[\frac{1}{2}, 1\right] \times[-1,1] \rightarrow S^{1} \times\left[\frac{1}{2}, 1\right] \times[-1,1] .
\end{aligned}
$$

We must have $\left(\left.\iota_{-}\right|_{S \times \frac{1}{2} \times[-1,1]}\right)_{*}=-\left(\left.\iota_{+}\right|_{S \times \frac{1}{2} \times[-1,1]}\right)_{*}$ and we conclude that exactly one of $\left(\iota_{+}\right)_{*},\left(\iota_{-}\right)_{*}$ is -id on the first homology group. Hence, exactly one of $\left(\left.\iota\right|_{V_{i}}\right)_{*}$ : $H_{1}\left(V_{i}\right) \rightarrow H_{1}\left(V_{i}\right), i=+,-$, must be -id. By Lemma (2.3), exactly one of $\left.\iota\right|_{V_{i}}$ : $V_{i} \rightarrow V_{i}, i=+,-$, will have 2 fixed points, and the other will be fixed point free.

(4) Suppose $\iota$ has a fixed point. We show that there is a nonseparating proper annulus $A \subset W$ with $A \cap \iota(A)=\varnothing$.

By (1) and (2), let $A_{0} \subset W$ be a nonseparating proper annulus with either $A_{0} \cap \iota\left(A_{0}\right)=\varnothing$ or $\iota\left(A_{0}\right)=A_{0}$ and with no fixed points of $\iota$ on $A_{0}$ in the latter case.

If $A_{0} \cap \iota\left(A_{0}\right)=\varnothing$, let $A=A_{0}$.

Now assume $\iota\left(A_{0}\right)=A_{0}$. By Lemma (2.1), let $A_{0} \times[-\varepsilon, \varepsilon]$ be a bicollar of $A_{0}=A_{0} \times 0$ in $W$ with $\left(A_{0} \times[-\varepsilon, \varepsilon]\right) \cap \partial W=\partial A_{0} \times[-\varepsilon, \varepsilon], \iota\left(A_{0} \times[-\varepsilon, \varepsilon]\right)=A_{0}$ $\times[-\varepsilon, \varepsilon]$, and with no fixed points of $\iota$ on $A_{0} \times[-\varepsilon, \varepsilon]$. 
If $\iota\left(A_{0} \times \varepsilon\right)=A_{0} \times-\varepsilon$, let $A=A_{0} \times \varepsilon$.

Lastly suppose that $\iota\left(A_{0} \times \varepsilon\right)=A_{0} \times \varepsilon$. For the following argument we may assume that $A_{0} \times \varepsilon=A_{*}$. Let $V=\overline{W-A_{0} \times[-\varepsilon, \varepsilon]}$. Then $V$ is a solid torus and the inclusion $j: A \times \varepsilon \rightarrow V$ induces an isomorphism $j_{*}: H_{1}(A \times \varepsilon) \rightarrow H_{1}(V)$. The involution $\iota \mid: V \rightarrow V$ has a fixed point. By Lemma (2.3), then, $\left(\left.\iota\right|_{V}\right)_{*}: H_{1}(V) \rightarrow H_{1}(V)$ is $\left(\left.\iota\right|_{V}\right)_{*}=-\mathrm{id}$. On the other hand, since $\iota \mid: A \times \varepsilon \rightarrow A \times \varepsilon$ is fixed point free, we must have $(\iota \mid)_{*}: H_{1}(A \times \varepsilon) \rightarrow H_{1}(A \times \varepsilon)$ is $(\iota \mid)_{*}=$ id. By commutativity with respect to $j_{*}$ it follows that $\left(\left.\iota\right|_{V}\right)_{*}=\mathrm{id}$. This contradiction proves that $\iota(A \times \varepsilon)=A$ $\times \varepsilon$ is not possible. Q.E.D.

We now introduce canonical generators of $H_{1}(\partial W)$ and $H_{1}(W)$.

Define $S_{0}=q\left(S^{1} \times 0 \times 1\right)=q\left(S^{1} \times 1 \times-1\right) \subset \partial W, \quad S_{1}=q(1 \times[0,1] \times 0) \subset$ $W$, and $S_{2}=q(1 \times[0,1] \times 1) \cup q(1 \times[0,1] \times-1) \subset \partial W$. Then

$$
H_{1}(\partial W)=\mathbf{Z}\left[S_{0}\right] \oplus \mathbf{Z}\left[S_{2}\right] \text { and } H_{1}(W)=\mathbf{Z}_{2}\left[S_{0}\right] \oplus \mathbf{Z}\left[S_{1}\right] .
$$

Let $j: \partial W \rightarrow W$ be the inclusion. Then $j_{*}\left[S_{0}\right]=\left[S_{0}\right]$ and $j_{*}\left[S_{2}\right]=2\left[S_{1}\right]$.

Note that $H_{i}(W)=H_{i}(K)=0, i \geqslant 2$.

Now let $\iota: W \rightarrow W$ be an involution and consider $(\iota \mid)_{*}: H_{1}(\partial W) \rightarrow H_{1}(\partial W)$.

PROPOSITION (4.5). (I) If $\iota$ is orientation preserving, then $(\iota \mid)_{*}=\mathrm{id}$.

(II) If $\iota$ is orientation reversing without fixed points, then

$$
(\iota \mid)_{*}\left[S_{0}\right]=-\left[S_{0}\right], \quad(\iota \mid)_{*}\left[S_{2}\right]=\left[S_{2}\right]+k\left[S_{0}\right] .
$$

(III) If $\iota$ is orientation reversing with fixed points, then

$$
(\iota \mid)_{*}\left[S_{0}\right]=\left[S_{0}\right], \quad(\iota \mid)_{*}\left[S_{2}\right]=-\left[S_{2}\right]+l\left[S_{0}\right] .
$$

Proof. By Main Lemma (4.4), there is a nonseparating proper annulus $A \subset W$ with either $A \cap \iota(A)=\varnothing$ or with $\iota(A)=A$. By Corollary (4.3) we may assume that $A=A_{*}$. We conclude that

$$
(\iota \mid)_{*}\left[S_{0}\right]= \pm\left[S_{0}\right]
$$

Consider $\iota_{*}: H_{1}(W) \rightarrow H_{1}(W)$. If $\iota$ is fixed point free, then its Lefschetz number must be 0 , and therefore $\iota_{*}=$ id. If $\iota$ has fixed points, then by Lemma (2.3) its Lefschetz number must be 2 , and hence $\iota_{*}=-$ id. It follows from commutativity that $(\iota \mid)_{*}\left[S_{2}\right]=\left[S_{2}\right]+k\left[S_{0}\right]$ if $\iota$ is fixed point free, and that $(\iota \mid)_{*}\left[S_{2}\right]=-\left[S_{2}\right]+l\left[S_{0}\right]$ if $\iota$ has fixed points.

If $\iota$ is orientation preserving so is $\left.\iota\right|_{\partial W}$ and we must have $(\iota \mid)_{*}\left[S_{0}\right]=\left[S_{0}\right]$. Since $(\iota \mid)_{*}^{2}=$ id, we conclude that $(\iota \mid)_{*}\left[S_{2}\right]=\left[S_{2}\right]$, i.e. $(\iota \mid)_{*}=$ id.

If $\iota$ is orientation reversing so is $\left.\iota\right|_{\partial W}$. Therefore $(\iota)_{*}\left[S_{0}\right]=-\left[S_{0}\right]$ if $\iota$ is fixed point free, and $(\iota \mid)_{*}\left[S_{0}\right]=\left[S_{0}\right]$ if $\iota$ has fixed points. Q.E.D.

LEMMA (4.6). Let $A \subset W$ be a nontrivial proper annulus with one component $S^{\prime}$ of $\partial A$ satisfying $\left[S^{\prime}\right]=\left[S_{0}\right]$ in $H_{1}(\partial W)$. Then $A$ is nonseparating.

Proof. We may assume that $S^{\prime}=S_{0}$.

Consider the proper Moebius strip $M O=q(1 \times[0,1] \times[-1,1]) \subset W$. Then $\partial M O$ and $S_{0}$ intersect transversally in exactly one point $q(1 \times 1 \times 1)$. Applying an ambient isotopy to $A$ keeping $S_{0}$ pointwise fixed, we may assume that $A$ and $M O$ 
intersect transversally in a single 1-cell $I$, which is proper in both $A$ and $M O$. It must meet both components of $\partial A$. Since $A$ is nontrivial, it cannot separate $M O$. Hence $A$ does not separate $W$. Q.E.D.

THEOREM (4.7). Suppose $\iota, \iota^{\prime} W \rightarrow W$ are involutions and one of the following holds:

(I) $\iota, \iota^{\prime}$ are orientation preserving,

(II) $\iota, \iota$ are orientation reversing without fixed points, or

(III) $\iota, \iota^{\prime}$ are orientation reversing with fixed points.

Then there is an isomorphism $h: W \rightarrow W$ so that $\iota^{\prime}=h \iota^{-1}$.

Moreover, in case (III), if $h_{0}: \partial W \rightarrow \partial W$ is an isomorphism with $\left.\iota^{\prime}\right|_{\partial W}=h_{0} \iota h_{0}^{-1}$, we may assume that $\left.h\right|_{\partial W}=h_{0}$.

Proof. (1) Suppose $\iota, \iota^{\prime}$ have no fixed points. By Main Lemma (4.4), there is a nonseparating proper annulus $A \subset W$ with $\iota(A)=A$. By Lemma (2.1), let $A \times$ $[-\varepsilon, \varepsilon] \subset W$ be a bicollar of $A=A \times 0$ in $W$ with $A \times[-\varepsilon, \varepsilon] \cap \partial W=\partial A \times[-\varepsilon, \varepsilon]$ and $\iota(A \times[-\varepsilon, \varepsilon])=A \times[-\varepsilon, \varepsilon]$. By Main Lemma (4.4), $\iota(A \times i \varepsilon)=A \times i \varepsilon, i= \pm$.

For the following compare the proof of lemma (3.7).

Let $I \subset A$ be a nonseparating proper 1-cell with $I \cap \iota(I)=\varnothing$. We may assume that $\varepsilon$ is sufficiently small so that $(I \times[-\varepsilon, \varepsilon]) \cap \iota(I \times[-\varepsilon, \varepsilon])=\varnothing$.

By Corollary (4.3) we may assume $A=A_{*}$. Hence $V=\overline{W-A \times[-\varepsilon, \varepsilon]}$ is a solid torus. By the argument in Lemma (3.7), there is a nonseparating proper 2-cell $B \subset V$ with $B \cap A \times i \varepsilon=I \times i \varepsilon, i= \pm$, and with $B \cap \iota(B)=\varnothing$.

$I \times[-\varepsilon, \varepsilon] \cup \iota(I \times[-\varepsilon, \varepsilon])$ decomposes $A \times[-\varepsilon, \varepsilon]$ into the 3-cells $C, \iota(C)$ :

$$
A \times[-\varepsilon, \varepsilon]=C \cup \iota(C) \text { and } C \cap \iota(C)=I \times[-\varepsilon, \varepsilon] \cup \iota(I \times[-\varepsilon, \varepsilon]) \text {. }
$$

$B \cup \iota(B)$ decomposes $V$ into the 3-cells $E, \iota(E)$ :

$$
V=E \cup \iota(E) \text { and } E \cap \iota(E)=B \cup \iota(B) .
$$

We may assume that $E \cap A \times \varepsilon=C \cap A \times \varepsilon$.

Similarly, $A^{\prime}, A^{\prime} \times\left[-\varepsilon^{\prime}, \varepsilon^{\prime}\right], I^{\prime}, V^{\prime}, B^{\prime}, C^{\prime}$, and $E^{\prime}$ are defined with respect to $\iota^{\prime}$.

Let $\partial I=\left\{x_{0}, x_{1}\right\}, \partial I^{\prime}=\left\{x_{0}^{\prime}, x_{1}^{\prime}\right\}$. Choose an arbitrary isomorphism

$$
\begin{aligned}
h_{0}: & \left(I \times[-\varepsilon, \varepsilon], x_{0} \times[-\varepsilon, \varepsilon], x_{1} \times[-\varepsilon, \varepsilon], I \times-\varepsilon, I \times \varepsilon\right) \\
& \rightarrow\left(I^{\prime} \times\left[-\varepsilon^{\prime}, \varepsilon^{\prime}\right], x_{0}^{\prime} \times\left[-\varepsilon^{\prime}, \varepsilon^{\prime}\right], x_{1}^{\prime} \times\left[-\varepsilon^{\prime}, \varepsilon^{\prime}\right], I^{\prime} \times-\varepsilon^{\prime}, I^{\prime} \times \varepsilon^{\prime}\right) .
\end{aligned}
$$

Define $h_{1}: \iota(I \times[-\varepsilon, \varepsilon]) \rightarrow \iota^{\prime}\left(I^{\prime} \times\left[-\varepsilon^{\prime}, \varepsilon^{\prime}\right]\right)$ by equivariance, $h_{1}=\left.\iota^{\prime} h_{0} \iota\right|_{\iota(I \times[-\varepsilon, \varepsilon])}$. Since $\iota, \iota^{\prime}$ have the same orientation type, the isomorphism

$$
h_{0} \cup h_{1}: I \times[-\varepsilon, \varepsilon] \cup \iota(I \times[-\varepsilon, \varepsilon]) \rightarrow I^{\prime} \times\left[-\varepsilon^{\prime}, \varepsilon^{\prime}\right] \cup \iota^{\prime}\left(I^{\prime} \times\left[-\varepsilon^{\prime}, \varepsilon^{\prime}\right]\right)
$$

extends to an isomorphism

$$
\begin{aligned}
h_{2}: & (C, I \times[-\varepsilon, \varepsilon], \iota(I \times[-\varepsilon, \varepsilon]), C \cap A \times \varepsilon, C \cap A \times-\varepsilon) \\
& \rightarrow\left(C^{\prime}, I^{\prime} \times\left[-\varepsilon^{\prime}, \varepsilon^{\prime}\right], \iota^{\prime}\left(I^{\prime} \times\left[-\varepsilon^{\prime}, \varepsilon^{\prime}\right]\right), C^{\prime} \cap A^{\prime} \times \varepsilon^{\prime}, C^{\prime} \cap A^{\prime} \times-\varepsilon^{\prime}\right) .
\end{aligned}
$$

The isomorphism $h_{0} \mid:(I \times \varepsilon, I \times-\varepsilon) \rightarrow\left(I^{\prime} \times \varepsilon^{\prime}, I^{\prime} \times-\varepsilon^{\prime}\right)$ extends to an isomorphism $h_{3}: B \rightarrow B^{\prime}$, and the isomorphism

$$
\begin{aligned}
& h_{3} \cup h_{2} \mid: B \cup(E \cap A \times \varepsilon) \cup(E \cap A \times-\varepsilon) \\
& \rightarrow B^{\prime} \cup\left(E^{\prime} \cap A^{\prime} \times \varepsilon^{\prime}\right) \cup\left(E^{\prime} \cap A^{\prime} \times-\varepsilon^{\prime}\right)
\end{aligned}
$$


extends to an isomorphism

$$
h_{4}:(E, B, \iota(B)) \rightarrow\left(E^{\prime}, B^{\prime}, \iota^{\prime}\left(B^{\prime}\right)\right) .
$$

The isomorphisms $h_{2}$ and $h_{4}$ piece together.

Finally, by equivariance define

$$
h=\left.h_{2} \cup h_{4} \cup \iota^{\prime}\left(h_{2} \cup h_{4}\right) \iota\right|_{\iota(C) \cup \iota(E)}: W \rightarrow W .
$$

Then $h$ is a well-defined isomorphism satisfying $\iota^{\prime}=h \iota h^{-1}$.

(2) Suppose $\iota, \iota^{\prime}$ have fixed points. Since $\partial W / \imath\left|, \partial W / \iota^{\prime}\right|$ are Klein bottles there exists an isomorphism $h_{0}: \partial W \rightarrow \partial W$ so that $\left.\iota^{\prime}\right|_{\partial W}=h_{0} \iota h_{0}^{-1}$. Thus we may assume that $h_{0}$ is given.

By Main Lemma (4.4) there is a nonseparating proper annulus $A \subset W$ with $A \cap \iota(A)=\varnothing$. By Corollary (4.3) we may assume that $A=A_{*}$ and $\iota(A)=A_{c}$. We make the following observations:

There are no annuli $A_{1}, A_{2} \subset \partial W$ with $\partial A_{1}=\partial A, \partial A_{2}=\partial \iota(A)$, and with $A_{1} \cap A_{2}$ $=\varnothing$.

Now $A \cup \iota(A)$ decomposes $W$ into 2 solid tori $V_{ \pm}$:

$$
W=V_{+} \cup V_{-} \text {and } V_{+} \cap V_{-}=\partial V_{+} \cap \partial V_{-}=A \cup \iota(A) \text {. }
$$

By Proposition $(4.5),(\iota \mid)_{*}\left[S_{0}\right]=\left[S_{0}\right],(\iota \mid)_{*}\left[S_{2}\right]=-\left[S_{2}\right]+l\left[S_{0}\right]$ and $\left(\iota^{\prime} \mid\right)_{*}\left[S_{0}\right]=\left[S_{0}\right]$, $\left(\iota^{\prime}\right)_{*}\left[S_{2}\right]=-\left[S_{2}\right]+l^{\prime}\left[S_{0}\right]$. The same computation as in part (1) of the proof of Theorem (3.3) gives

$$
\left(\left.h_{0}\right|_{\partial W}\right)_{*}\left[S_{0}\right]= \pm\left[S_{0}\right]
$$

Therefore there is an ambient isotopy on $W$ that maps $h_{0}\left(S_{0}\right)$ to $S_{0}$.

Consider next $h_{0}(\partial A)$ and $h_{0}(\iota \partial A)=\iota^{\prime} h_{0} \partial A$ in $W$. We have $h_{0}(\partial A) \cap \iota^{\prime} h_{0}(\partial A)=$ $h_{0}(\partial A \cap \iota \partial A)=\varnothing$.

Let $A_{0} \subset W$ be a nontrivial proper annulus with $A_{0} \cap \partial W=\partial A_{0}=h_{0}(\partial A)$. By Theorem (2.5) there is a nontrivial proper annulus $A^{\prime} \subset W$ with either

$$
A^{\prime} \cap \iota^{\prime}\left(A^{\prime}\right)=\varnothing \quad \text { and } \quad \partial A^{\prime} \cup \iota^{\prime}\left(\partial A^{\prime}\right)=\partial A_{0} \cup \iota^{\prime}\left(\partial A_{0}\right)
$$

or there are 2 disjoint nontrivial proper annuli $\tilde{A}_{1}, \tilde{A}_{2}$ in $W$ with $\iota^{\prime}\left(\tilde{A}_{i}\right)=\tilde{A}_{i}$, $i=1,2 ; \partial\left(\tilde{A}_{1} \cup \tilde{A}_{2}\right)=\partial A_{0} \cup \iota^{\prime}\left(\partial A_{0}\right)$; and with no fixed points of $\iota^{\prime}$ on $\tilde{A}_{1} \cup \tilde{A}_{2}$.

By Lemma (4.6), $A^{\prime}, \tilde{A}_{1}, \tilde{A}$, are all nonseparating.

The second case cannot occur. (Namely otherwise by Corollary (4.3), we may assume that $\tilde{A}_{1}=A_{*}, \tilde{A}_{2}=A$. We have the decomposition $W=\tilde{V}_{+} \cup \tilde{V}_{-}, \tilde{V}_{ \pm}$solid tori with $\tilde{V}_{+} \cap \tilde{V}_{-}=\partial \tilde{V}_{+} \cap \partial \tilde{V}_{-}=\tilde{A}_{1} \cup \tilde{A}_{2}$. Necessarily $\iota^{\prime}\left(\tilde{V}_{i}\right)=\tilde{V}_{i}, i= \pm$. The inclusion $j_{i}: \tilde{A}_{1} \rightarrow \tilde{V}_{i}$ induces an isomorphism $j_{i *}: H_{1}\left(\tilde{A}_{1}\right) \rightarrow H_{1}\left(\tilde{V}_{1}\right)$. Since $\iota^{\prime} \mid: \tilde{A}_{1} \rightarrow \tilde{A_{1}}$ is fixed point free, we must have $\left(\iota^{\prime} \mid\right)_{*}=\mathrm{id}$. By commutativity $\left(\iota^{\prime} \mid \tilde{V}_{i}\right)_{*}=\mathrm{id}$ and the Lefschetz number of $\iota^{\prime} \mid \tilde{V}_{i}$ is 0 . By Lemma (2.3), $\iota^{\prime} \mid \tilde{V}_{i}$ has no fixed points, $i= \pm$, and hence $\iota^{\prime}$ is fixed point free. Contradiction.)

Therefore there is a nonseparating proper annulus $A^{\prime} \subset W$ with $A^{\prime} \cap \iota^{\prime}\left(A^{\prime}\right)=\varnothing$ and $\partial A^{\prime} \cup \iota^{\prime}\left(\partial A^{\prime}\right)=\partial A_{0} \cup \iota^{\prime}\left(\partial A_{0}\right)$.

It is not possible that one component of $\partial A^{\prime}$ is in $\partial A_{0}$ and the other is in $\iota^{\prime}\left(\partial A_{0}\right)$. Namely, otherwise there are annuli $A_{1}^{\prime}, A_{2}^{\prime} \subset \partial W$ with $\partial A_{1}^{\prime}=\partial A_{0}, \partial A_{2}^{\prime}=\iota^{\prime} \partial A_{0}$, and with $A_{1}^{\prime} \cap A_{2}^{\prime}=\varnothing$. Then $A_{i}=h_{0}^{-1}\left(A_{i}^{\prime}\right), i=1,2$, contradicting our observation. 
Thus we have $\partial A^{\prime}=\partial A_{0}=h_{0}(\partial A)$ and hence $\iota^{\prime}\left(\partial A^{\prime}\right)=h_{0} \iota \partial A$.

Again $A^{\prime} \cup \iota^{\prime}\left(A^{\prime}\right)$ decomposes $W$ into 2 solid tori $V_{ \pm}^{\prime}$ :

$$
W=V_{+}^{\prime} \cup V_{-}^{\prime} \text { and } V_{+}^{\prime} \cap V_{-}^{\prime}=\partial V_{+}^{\prime} \cap \partial V_{-}^{\prime}=A^{\prime} \cup \iota^{\prime}\left(A^{\prime}\right) .
$$

The isomorphism $h_{0} \mid: \partial A \rightarrow \partial A^{\prime}$ can be extended to an isomorphism $\hat{h}_{0}: A \rightarrow A^{\prime}$. Define the isomorphism

$$
h_{i}=\left.\left.\hat{h}_{0} \cup h_{0}\right|_{V_{i} \cap \partial W} \cup \iota^{\prime} \hat{h}_{0} \iota\right|_{\iota(A)}: \partial V_{i} \rightarrow \partial V_{i}^{\prime}, \quad i= \pm .
$$

By construction $\left.\iota^{\prime}\right|_{\partial V^{\prime}}=h_{i} \iota h_{i}^{-1}, i= \pm$. By Theorem (3.3), $h_{i}$ extends to an isomorphism $\hat{h}_{i}: V_{i} \rightarrow V_{i}^{\prime}$ with $\left.\iota^{\prime}\right|_{V_{i}^{\prime}}=\hat{h}_{i} \iota \hat{h}_{i}^{-1}, i= \pm$. Finally, define $h=\hat{h}_{+} \cup \hat{h}_{-}: W \rightarrow W$. Then $h$ is a well-defined isomorphism extending $h_{0}$ and satisfying $\iota^{\prime}=h \iota h^{-1}$. Q.E.D.

Corollary (4.8). Suppose $\iota: W \rightarrow W$ is an involution. Then $\iota$ is conjugate to:

(I) $\nu$, if $\iota$ is orientation preserving,

(II) $\rho_{0}$, if $\iota$ is orientation reversing and fixed point free, or

(III) $\rho_{2}$, if $\iota$ is orientation reversing with fixed points. Moreover, in case (III), if $h_{0}$ : $\partial W \rightarrow \partial W$ is an isomorphism with $\left.\iota\right|_{\partial W}=h_{0} \rho_{2} h_{0}^{-1}$, then $h_{0}$ extends to an isomorphism $h: W \rightarrow W$ with $\iota=h \rho_{2} h^{-1}$.

Let $M O$ be a Moebius strip and let $\psi: M O \rightarrow M O$ be an involution with fixed set an isolated point and a proper 1-cell. Then $M O \times[0,1] /(x, 0) \sim(\psi(x), 1)$ is the nonorientable twisted I-bundle over a Klein bottle.

Corollary (4.9). Suppose $\iota: W \rightarrow W$ is a fixed point free involution.

(I) If $\iota$ is orientation preserving, then $W / \iota=W$.

(II) If $\iota$ is orientation reversing, then $W / \iota=$ nonorientable twisted I-bundle over a Klein bottle.

Proof. By Corollary (4.8), we may assume that $W / \iota=W / \nu$ in case (I) and that $W / \iota=W / \rho_{0}$ in case (II). The corollary is easily verified for $W / \nu$ and for $W / \rho_{0}$. Q.E.D.

5. Involutions with fixed points on orientable torus bundles over the 1-sphere. Let $S_{1}, S_{2}$ be copies of the 1-sphere and let $T=S_{1} \times S_{2}$ be the 2-dimensional torus. If $g$ : $T \rightarrow T$ is an isomorphism then the associated torus bundle $M_{g}$ over the 1-sphere is defined as follows:

$$
M_{g}=T \times[0,1] /(x, 0) \sim(g(x), 1) .
$$

Let $q: T \times[0,1] \rightarrow M_{g}$ be the identification map and abbreviate $q(x, y, t)=$ $[x, y, t]$. Let $S^{1}=[0,1] / 0 \sim 1$. Define $\pi: M_{g} \rightarrow S^{1}$ by $\pi[x, y, t]=t$. Note that each fiber $\pi^{-1}(t)=q(T \times t)$ is a nonseparating, 2-sided, incompressible torus. Clearly $M_{g}$ is irreducible.

Every torus bundle over $S^{1}$ arises this way.

The isomorphism $g: T \rightarrow T$ is up to isotopy uniquely determined by the isomorphism $g_{*}: H_{1}(T) \rightarrow H_{1}(T)$. With respect to the basis $\left[S_{1}\right],\left[S_{2}\right]$ of $H_{1}(T)=\mathbf{Z} \oplus \mathbf{Z}$, $g_{*}$ is given by a matrix $A \in \mathrm{GL}_{2}(\mathbf{Z})$. The torus bundle $M_{g}$ is orientable if, and only if, the isomorphism $g: T \rightarrow T$ is orientation preserving. This is equivalent to $\operatorname{det} A=1$. 
It is also possible to have separating incompressible tori in $M_{g}$. As an example of this consider the space form $M_{2}$ (see [7,8]). It is the torus bundle corresponding to $g(x, y)=(\bar{x}, \bar{y})$. The annuli $A_{ \pm}=\{ \pm i\} \times S_{2} \times[0,1]$ piece together to give the separating incompressible torus $q\left(A_{+} \cup A_{-}\right)$. Cutting $M_{2}$ along this torus then gives 2 copies of $W=$ the oriented twisted $I$-bundle over the Klein bottle.

The main result of this section is the complete classification of involutions with fixed points on orientable torus bundles over $S^{1}$. It turns out that only $M_{1}=S^{1} \times$ $S^{1} \times S^{1}$ and $M_{2}$ admit such involutions, and moreover they are unique up to conjugacy.

The unique involution on $M_{1}$ is $\iota_{8}: M_{1} \rightarrow M_{1}, \iota_{8}\left(x_{1}, x_{2}, x_{3}\right)=\left(\bar{x}_{1}, \bar{x}_{2}, \bar{x}_{3}\right)$. It has 8 fixed points $( \pm 1, \pm 1, \pm 1)$.

The unique involution on $M_{2}$ is given by

$$
\iota_{4}[x, y, t]= \begin{cases}{\left[-x,-y, \frac{1}{2}-t\right]} & \text { if } 0 \leqslant t \leqslant \frac{1}{2}, \\ {\left[-\bar{x},-\bar{y}, \frac{3}{2}-t\right]} & \text { if } \frac{1}{2} \leqslant t \leqslant 1 .\end{cases}
$$

There are 4 fixed points $\left[ \pm i, \pm i, \frac{3}{4}\right]$.

Lemma (5.1). Suppose $T_{0} \subset$ int $T \times[0,1]$ is an incompressible torus. Then there is an ambient isotopy of $T \times[0,1]$ taking $T_{0}$ onto $T \times \frac{1}{2}$ and leaving $\partial(T \times[0,1])$ pointwise fixed.

Proof. Applying an ambient isotopy to $T_{0}$ keeping the boundary pointwise fixed, we may assume that $T_{0} \cap\left(S_{1} \times y_{0} \times[0,1]\right)=S_{1} \times y_{0} \times \frac{1}{2}$ and then that

$$
T_{0} \cap\left(x_{0} \times S_{2} \times[0,1]\right)=x_{0} \times S_{2} \times \frac{1}{2} .
$$

Now there is an ambient isotopy that keeps the boundary and $S_{1} \times y_{0} \times[0,1] \cup x_{0}$ $\times S_{2} \times[0,1]$ pointwise fixed, and maps $T_{0}$ to $T \times \frac{1}{2}$. Q.E.D.

LEMMA (5.2). Let $W$ be the orientable twisted I-bundle over a Klein bottle. If $T \subset$ int $W$ is an incompressible torus, then there is a collar $\partial W \times[0,1]$ of $\partial W=\partial W \times 0$ in $W$ so that $\partial W \times 1=T$.

Proof. Let $p: \tilde{W} \rightarrow W$ be the 2-fold covering with $p_{*} \pi_{1}(\tilde{W})=j_{*} \pi_{1}(\partial W)$, where $j: \partial W \rightarrow W$ is the inclusion. Then $\tilde{W}=\partial \tilde{W} \times[0,3], p \mid: \partial \tilde{W} \times i \rightarrow \partial W$ is an isomorphism, $i=0,3$, and $p^{-1}(T)$ must consist of 2 components $T_{1}$ and $T_{2}$, each an incompressible torus. By Lemma (5.1) we may assume that $T_{1}=\partial \tilde{W} \times 1$ and that $T_{2}=\partial \tilde{W} \times 2$. Let $\partial W \times[0,1]=p(\partial \tilde{W} \times[0,1])$. Q.E.D.

Lemma (5.3). Let $M$ be an orientable torus bundle over $S^{1}$ and let $T \subset M$ be an incompressible torus.

(1) Suppose $T$ is nonseparating. Then $\overline{M-T \times[-1,1]}$, where $T \times[-1,1]$ is a bicollar of $T=T \times 0$ in $M$, is another copy of $T \times[-1,1]$.

(2) Suppose $T$ is separating. Then $T$ separates $M$ into orientable twisted I-bundles $W_{1}, W_{2}$ over a Klein bottle. Moreover, there are nonseparating proper annuli $A_{i *} \subset W_{i}$, $i=1,2$, so that $A_{1 *} \cap A_{2 *}$ is a boundary component of both $A_{1 *}$ and $A_{2 *}$. 
Proof. First note that $T$ is 2 -sided and so has a bicollar $T \times[-1,1]$. Also $\overline{M-T \times[0,1]}$ will have 1 or 2 components.

Let $T_{*}=q(T \times 0)$ be a distinguished fiber. Applying an ambient isotopy to $T$ we may assume that $T$ and $T_{*}$ intersect transversally in disjoint 1 -spheres $S_{1}, \ldots, S_{n}$. Since $M$ is irreducible, by standard arguments we may assume that none of the 1-spheres $S_{1}, \ldots, S_{n}$ bound 2-cells in $T$ or $T_{*}$. If $T \cap T_{*}=\varnothing$, then Lemma (5.1) implies that case (1) holds.

Now assume that $n=1$. Then $q^{-1}(T) \subset T \times[0,1]$ is an incompressible proper annulus meeting both boundary components. By Lemma (3.6) $\left(T \times[0,1], q^{-1}(T)\right)$ is isomorphic to $\left(S^{1} \times S^{1} \times[0,1], x_{0} \times S^{1} \times[0,1]\right)$. Let $T \times[-1,1] \subset M$ be a bicollar of $T=T \times 0$ in $M$ with $(T \times[-1,1]) \cap T_{*}=S_{*}^{1} \times[-1,1]$. Then we may assume that $q^{-1}(T \times[-1,1])=I \times S^{1} \times[0,1]$ where $I \subset S^{1}$ is a 1-cell with $x_{0} \in$ int $I$ and with $q\left(I \times S^{1} \times 0\right)=q\left(I \times S^{1} \times 1\right)$. Since $\overline{M-T \times[0,1]}$ has two boundary components and is connected we conclude that

$$
\overline{M-T \times[0,1]}=q\left(\overline{S^{1}-I} \times S^{1} \times[0,1]\right)=T \times[-1,1] .
$$

Finally suppose that $n>1$. Now the 1 -spheres $S_{1}, \ldots, S_{n}$ decompose $T$ into annuli $A_{1}, \ldots, A_{n}$. We arrange the ordering so that $\partial A_{i}=S_{i} \cup S_{i+1}, i=1, \ldots, n$, where $S_{n+1}=S_{1}$.

Consider the proper annuli $B_{i}=\overline{q^{-1}\left(\text { int } A_{i}\right)} \subset T \times[0,1], i=1, \ldots, n$.

If an annulus $B_{i}$ has both boundary components in the same boundary component of $T \times[0,1]$ then there is a solid torus $V \subset T \times[0,1]$ with $B_{i} \subset \partial V, V \cap$ $\partial(T \times[0,1])=\overline{\partial V-B_{i}}$, and such that there is a nonseparating proper 2-cell $B \subset V$ with $B \cap B_{i}$ a 1-cell. Using $q(V) \subset M$, we can construct an ambient isotopy on $M$ which applied to $T$ will remove $S_{i}$ and $S_{i+1}$ from $T \cap T_{*}$. Thus we may assume that each annulus $B_{i} \subset T \times[0,1]$ meets both boundary components of $T \times[0,1]$.

Again by Lemma (3.6) $\left(T \times[0,1], B_{1}, \ldots, B_{n}\right)$ is isomorphic to $\left(S^{1} \times S^{1} \times\right.$ $\left.[0,1], x_{1} \times S^{1} \times[0,1], \ldots, x_{n} \times S^{1} \times[0,1]\right)$. Let $T \times[-1,1]$ be a bicollar of $T=T$ $\times 0$ in $M$ with $T \times[-1,1] \cap T_{*}=S_{i} \times[-1,1], i=1, \ldots, n$. Then $\overline{q^{-1}\left(\text { int } A_{i} \times[-1,1]\right)}=I_{i} \times S^{1} \times[0,1]$, where $I_{1}, \ldots, I_{n} \subset S^{1}$ are disjoint 1-cells with $x_{i} \in$ int $I_{i}$ and $q\left(I_{i} \times S^{1} \times 1\right)=q\left(I_{i+1} \times S^{1} \times 0\right)$, and where $I_{n+1}=I_{1}$. It follows that

$$
\overline{M-T \times[-1,1]}=\bigcup_{i=1}^{n} q\left(J_{i} \times S^{1} \times[0,1]\right),
$$

where the $J_{i} \subset S^{1}$ are the complementary intervals determined by $I_{1} \cup \cdots \cup I_{n}$ in $S^{1}$.

The components of $\bigcup_{i=1}^{n} q\left(J_{i} \times S^{1} \times[0,1]\right)$ are either copies of $T \times[-1,1]$ or $W$. If $T$ is nonseparating then it automatically follows that the only component of $\overline{M-T \times[-1,1]}$ is a copy of $T \times[-1,1]$. On the other hand if $T$ separates $M$, then the 2 components of $\overline{M-T \times[-1,1]}$ must necessarily be copies of $W$.

Finally, let $A_{1 *}, A_{2 *}$ be adjacent annuli of $W_{1} \cap T_{*}, W_{2} \cap T_{*}$, respectively. Q.E.D. 
MAIN Lemma (5.4). Let $M$ be an orientable torus bundle over the 1-sphere and let $\iota$ : $M \rightarrow M$ be an involution with fixed points. Then there is a nonseparating incompressible torus $T \subset M$ with $T \cap \iota(T)=\varnothing$.

Proof. By Theorem (2.7) there exists an incompressible torus $T_{0} \subset M$ so that either $T_{0} \cap \iota\left(T_{0}\right)=\varnothing$ or $\iota\left(T_{0}\right)=T_{0}$. In the latter case there are no fixed points of $\iota$ on $T_{0}$.

(1) Suppose $\iota\left(T_{0}\right)=T_{0}$ and $T_{0}$ is nonseparating. By Lemma (2.1), let $T_{0} \times[-1,1]$ be a bicollar so that $\iota\left(T_{0} \times[-1,1]\right)=T_{0} \times[-1,1]$ and $\iota$ has no fixed points on $T_{0} \times[-1,1]$. By Lemma (5.3) $\overline{M-T_{0} \times[-1,1]}=T_{1} \times[-1,1]$ and $\iota$ has a fixed point on $T_{1} \times[-1,1]$. Finally by Lemma (3.9) we must have $\iota\left(T_{1} \times 1\right)=T_{1} \times-1$. Consequently $T=T_{0} \times 1$ will satisfy the conclusion of the Main Lemma.

(2) Now suppose $T_{0} \cap \iota\left(T_{0}\right)=\varnothing$ and $T_{0}$ separates $M$.

By Lemma (5.3) we have $M=W_{1} \cup W_{2}^{\prime}$, where $W_{1}, W_{2}^{\prime}$ are copies of $W$ and $W_{1} \cap W_{2}^{\prime}=\partial W_{1}=\partial W_{2}^{\prime}=T_{0}$. Suppose $\iota\left(T_{0}\right) \subset W_{2}^{\prime}$. Then by Lemma (5.2) we have the following decomposition of $M: M=W_{1} \cup T^{\prime} \times[0,1] \cup W_{2}, W_{1}, W_{2}$ disjoint copies of $W$ so that $W_{1} \cap T^{\prime} \times[0,1]=\partial W_{1}=T^{\prime} \times 0$, and $W_{2} \cap T^{\prime} \times[0,1]=\partial W_{2}$ $=T^{\prime} \times 1$, where $T^{\prime} \times 1=\iota\left(T_{0}\right)$. Necessarily $\iota\left(W_{1}\right)=W_{2}$ and $\iota\left(T^{\prime} \times[0,1]\right)=T^{\prime} \times$ $[0,1]$.

Let $A_{0} \subset W_{1}$ be a nonseparating proper annulus and let $\partial A_{0}=S^{\prime} \cup S^{\prime \prime}$. Then there is a proper annulus $A^{\prime} \subset T^{\prime} \times[0,1]$ so that $\partial A^{\prime}=S^{\prime} \cup \iota\left(S^{\prime \prime}\right)$. Thus $\partial A^{\prime} \cap$ $\iota\left(\partial A^{\prime}\right)=\varnothing$. We apply Theorem (2.5): Either there is an incompressible proper annulus $A \subset T^{\prime} \times[0,1]$ meeting both boundary components with $A \cap \iota(A)=\varnothing$ and with $\partial A \cup \iota(\partial A)=\partial A^{\prime} \cup \iota\left(\partial A^{\prime}\right)$, or there are 2 disjoint incompressible annuli $A_{1}, A_{2} \subset T^{\prime} \times[0,1]$ meeting both boundary components with $\iota\left(A_{i}\right)=A_{i}, i=1,2$; $\partial\left(A_{1} \cup A_{2}\right)=\partial A^{\prime} \cup \iota\left(\partial A^{\prime}\right)$; and with no fixed points of $\iota$ on $A_{1} \cup A_{2}$.

The second alternative is not possible. Namely, by Lemma (3.9)

$$
(\iota \mid)_{*}: H_{1}\left(T^{\prime} \times[0,1]\right) \rightarrow H_{1}\left(T^{\prime} \times[0,1]\right)
$$

is $(\iota)_{*}=-$ id. By Lemma (3.6) we may assume that $A_{1}, A_{2}$ are in standard position. We conclude that $(\iota)_{*}: H_{1}\left(A_{i}\right) \rightarrow H_{1}\left(A_{i}\right)$ is also $(\iota \mid)_{*}=-$ id. Therefore the Lefschetz number of $\iota \mid: A_{i} \rightarrow A_{i}$ is 2 and $\iota \mid$ must have fixed points on $A_{i}$. A contradiction.

Thus we may assume that $A \cap \iota(A)=\varnothing$. Then $T_{1}=A_{0} \cup A \cup \iota\left(A_{0}\right) \cup \iota(A)$ is a nonseparating incompressible torus with $\iota\left(T_{1}\right)=T_{1}$ and with no fixed points of $\iota$ on $T_{1}$. We have arrived at case (1).

(3) Finally suppose that $\iota\left(T_{0}\right)=T_{0}$ and $T_{0}$ separates. By Lemma (5.3), we have the decomposition $M=W_{1} \cup W_{2}, W_{1}, W_{2} \subset M$ copies of $W$ with $W_{1} \cap W_{2}=\partial W_{1}=$ $\partial W_{2}=T_{0}$. Necessarily $\iota\left(W_{i}\right)=W_{i}, i=1,2$. Suppose that $\iota$ has fixed points in $W_{1}$. By Main Lemma 4.4 there is a nonseparating proper annulus $A_{1} \subset W_{1}$ with $A_{1} \cap \iota\left(A_{1}\right)=\varnothing$. It follows from Lemma (5.3) and from Corollary (4.3) that there is a nonseparating proper annulus $A_{2}^{\prime} \subset W_{2}$ with $\partial A_{2}^{\prime}=\partial A_{1}$. We apply Theorem (2.5) to $\iota \mid: W_{2} \rightarrow W_{2}$ with respect to $A_{2}^{\prime}$. By the same argument as in part (2) of the proof of Theorem (4.7) we obtain a nonseparating proper annulus $A_{2} \subset W_{2}$ with $\partial A_{2}=\partial A_{1}$ and with $A_{2} \cap \iota\left(A_{2}\right)=\varnothing$. Define $T=A_{1} \cup A_{2}$. Then $T$ is 2-sided. Therefore $T$ is a nonseparating incompressible torus with $T \cap \iota(T)=\varnothing$. Q.E.D. 
THEOREM (5.5). Let $M$ be an orientable torus bundle over $S^{1}$ and let $\iota: M \rightarrow M$ be an involution with fixed points. Then เ has exactly 4 or 8 fixed points.

Proof. Let $T \subset M$ be the torus of Main Lemma (5.4). Then Lemmas (5.1) and (5.3) imply that $M$ decomposes as $M=\left(T_{1} \times[0,1]\right) \cup\left(T_{2} \times[0,1]\right)$ with $\left(T_{1} \times[0,1]\right)$ $\cap\left(T_{2} \times[0,1]\right)=\partial\left(T_{1} \times[0,1]\right)=\partial\left(T_{2} \times[0,1]\right)=T \cup \iota(T)$. We must have

$$
\iota\left(T_{i} \times[0,1]\right)=T_{i} \times[0,1], \quad i=1,2 .
$$

By Corollary (3.11), $\iota$ has either 0 or 4 fixed points in $T_{i} \times[0,1], i=1,2$. Q.E.D.

Theorem (5.6). Let $M, M^{\prime}$ be orientable torus bundles over $S^{1}$ and let $\iota: M \rightarrow M, \iota^{\prime}$ : $M^{\prime} \rightarrow M^{\prime}$ be involutions with fixed points. If $\iota, \iota^{\prime}$ have the same number of fixed points then there exists an isomorphism $h: M \rightarrow M^{\prime}$ satisfying $\iota^{\prime}=h \iota h^{-1}$.

Proof. As in the proof of Theorem (5.5) we have decompositions $M=\left(T_{1} \times\right.$ $[0,1]) \cup\left(T_{2} \times[0,1]\right), M^{\prime}=\left(T_{1}^{\prime} \times[0,1]\right) \cup\left(T_{2}^{\prime} \times[0,1]\right)$ with respect to $\iota, \iota^{\prime}$, respectively. We may assume that $\iota \mid: T_{2} \times[0,1] \rightarrow T_{2} \times[0,1]$ and $\iota^{\prime} \mid: T_{2}^{\prime} \times[0,1] \rightarrow T_{2}^{\prime} \times$ $[0,1]$ have 4 fixed points each. Then $\iota \mid: T_{1} \times[0,1] \rightarrow T_{1} \times[0,1]$ and $\iota^{\prime} \mid: T_{1}^{\prime} \times[0,1]$ $\rightarrow T_{1}^{\prime} \times[0,1]$ have the same number of fixed points $(0$ or 4$)$.

By Theorem (3.10) there is an isomorphism $h_{1}: T_{1} \times[0,1] \rightarrow T_{1} \times[0,1]$ with $\left.\iota^{\prime}\right|_{T_{1}^{\prime} \times[0,1]}=h_{1} \iota h_{1}^{-1}$. Then

$$
\left.\iota^{\prime}\right|_{\partial\left(T_{2}^{\prime} \times[0,1]\right)}=\left.h_{1}\right|_{\partial\left(T_{2} \times[0,1]\right)} \iota\left(\left.h_{1}\right|_{\partial\left(T_{2} \times[0,1]\right)}\right) .
$$

By Theorem (3.10), there is an isomorphism $h_{2}: T_{2} \times[0,1] \rightarrow T_{2}^{\prime} \times[0,1]$ with $\left.\iota^{\prime}\right|_{T_{2}^{\prime} \times[0,1]}=h_{2} \iota h_{2}^{-1}$ and with $\left.h_{2}\right|_{\partial\left(T_{2} \times[0,1]\right)}=\left.h_{1}\right|_{\partial\left(T_{1} \times[0,1]\right)}$. Then the isomorphism $h=$ $h_{1} \cup h_{2}: M \rightarrow M^{\prime}$ satisfies $\iota^{\prime}=h \iota h^{-1}$. Q.E.D.

COROLlaRY 5.7. The only orientable torus bundles over $S^{1}$ admitting involutions with fixed points are $M_{1}, M_{2}$. Moreover, all such involutions on $M_{1}, M_{2}$ are conjugate to $\iota_{8}, \iota_{4}$, respectively.

6. Involutions with fixed points on unions along the boundaries of 2 orientable twisted $I$-bundles over Klein bottles. Let $W_{1}, W_{2}$ be copies of the orientable twisted $I$-bundle $W$ over a Klein bottle. If $g: \partial W_{1} \rightarrow \partial W_{2}$ is an isomorphism then the associated union $M_{g}$ of $W_{1}$ and $W_{2}$ is defined as follows:

$$
M_{g}=W_{1} \cup W_{2} / x \sim g(x) \text { for } x \in \partial W_{1} .
$$

Orientations on $W_{1}$ and $W_{2}$ can be chosen so that $M_{g}$ is orientable.

The manifold $M_{g}$ admits a 2 -fold covering by an orientable torus bundle over $S^{1}$. We observe that $M_{g}$ is irreducible.

We have $M_{\text {id }}=D(W)$, the double of $W$. It has a 2-fold covering by $S^{1} \times S^{1} \times S^{1}$. From this it can be deduced that $M_{\text {id }}=M_{2}$. Thus $D\left(\rho_{2}\right): D(W) \rightarrow D(W)$, the double of the involution $\rho_{2}: W \rightarrow W$ with 2 fixed points, is an involution with 4 fixed points on $M_{2}$.

The main result of this section is the complete classification of involutions with fixed points on unions along the boundaries of 2 orientable twisted $I$-bundles over Klein bottles. We prove that besides $M_{2}$ - which we have dealt with in $\$ 5$-only the 
space form $M_{6}$ admits such involutions. Furthermore, they are unique up to conjugacy.

The space form $M_{6}$, which is frequently called the Hantzsche-Wendt manifold in reference to [2], has the following description:

Let $\left[S_{0 i}\right],\left[S_{2 i}\right] \in H_{1}\left(\partial W_{i}\right), i=1,2$, be the canonical generators which were introduced in $\S 4$. Let $g_{0}: \partial W_{1} \rightarrow \partial W_{2}$ be an isomorphism so that $g_{0^{*}}\left[S_{01}\right]= \pm\left[S_{22}\right]$, $g_{0 *}\left[S_{21}\right]= \pm\left[S_{02}\right]$. Then $M_{6}=M_{g_{0}}$.

Let $\rho_{2}: W_{1} \rightarrow W_{1}$ be the involution with 2 fixed points and let $\rho_{0}: W_{2} \rightarrow W_{2}$ be the fixed point free orientation reversing involution. We may choose $g_{0}$ so that $\rho_{0} g_{0}=\left.g_{0} \rho_{2}\right|_{\partial W_{1}}$. Then $\iota_{2}=\rho_{2} \cup \rho_{0}: M_{6} \rightarrow M_{6}$ is a well-defined involution. It has 2 fixed points, namely those of $\rho_{2}$.

Now suppose $M$ is the union along the boundaries of 2 orientable $I$-bundles over Klein bottles.

Proposition (6.1). If $M$ contains a nonseparating incompressible torus, then $M$ is a torus bundle over $S^{1}$.

Proof. Let $p: \tilde{M} \rightarrow M$ be a 2 -fold covering of $M$ by a torus bundle $\tilde{M}$ over a 1-sphere. Let $T \subset M$ be a nonseparating incompressible torus. Then each component of $p^{-1}(T)$ is an incompressible torus in $\tilde{M}$.

(1) Suppose $p^{-1}(T)$ consists of 2 tori $T_{1}, T_{2}$. Then $T_{1}, T_{2}$ must be nonseparating. Otherwise, if one of $T_{1}, T_{2}$ is separating, by Lemmas (5.3) and (5.2) we obtain a decomposition of $\tilde{M}$ into 3 submanifolds. Since $p$ is a 2 -fold covering, we conclude that $T$ must be separating, a contradiction.

Thus by Lemmas (5.3) and (5.1) we have the decomposition $\tilde{M}=\left(T_{1} \times[0,1]\right) \cup$ $\left(T_{2} \times[0,1]\right)$ with $\left(T_{1} \times[0,1]\right) \cap\left(T_{2} \times[0,1]\right)=\partial\left(T_{1} \times[0,1]\right)=\partial\left(T_{2} \times[0,1]\right)=T_{1}$ $\cup T_{2}$. Then $M$ can be obtained from $T_{i} \times[0,1]$ by identifying $T_{i} \times 0$ with $T_{i} \times 1$, i.e. $M$ is a torus bundle over $S^{1}$.

(2) Suppose $p^{-1}(T)=\tilde{T}$ is a single torus. Let $T \times[-1,1] \subset M$ be a bicollar of $T=T \times 0$ in $M$. Then $p^{-1}(T \times[0,1])=\tilde{T} \times[-1,1]$ is a bicollar of $\tilde{T}=\tilde{T} \times 0$ in $\tilde{M}$. The deck transformation $\iota: \tilde{M} \rightarrow \tilde{M}$ is orientation preserving and we have $\iota(\tilde{T} \times[-1,1])=\tilde{T} \times[-1,1]$. As $\iota \mid: \tilde{T} \rightarrow \tilde{T}$ is orientation preserving, we must have $\iota(\tilde{T} \times i)=\tilde{T} \times i, i=1,-1$. Consequently $\tilde{T}$ is nonseparating. By Lemma (5.3), $\overline{\tilde{M}-\tilde{T} \times[-1,1]}=\tilde{T}^{\prime} \times[-1,1]$. By Corollary (3.12), $\overline{p(\tilde{M}-\tilde{T} \times[-1,1])}=T^{\prime} \times$ $[-1,1]$. Hence $M$ is a torus bundle over $S^{1}$. Q.E.D.

LEMMA (6.2). A separating incompressible torus in $M$ decomposes $M$ into 2 orientable twisted I-bundles over Klein bottles.

Proof. We proceed as in the proof of Proposition (6.1). Let $T \subset M$ be a separating incompressible torus.

(1) Suppose $p^{-1}(T)$ consists of 2 tori $T_{1}, T_{2}$. If one of the tori $T_{i}$ is separating, both are separating, and we obtain the decomposition $\tilde{M}=W_{1} \cup T \times[0,1] \cup W_{2}$, where $W_{1}, W_{2}$ are copies of $W$ with $\partial W_{1}=T \times 0=T_{1}$ and $\partial W_{2}=T \times 1=T_{2}$. Necessarily, $\iota(T \times 0)=T \times 1$ and hence $\iota\left(W_{1}\right)=W_{2}, \iota(T \times[0,1])=T \times[0,1]$. Then 
$M=p\left(W_{1}\right) \cup p(T \times[0,1])$ with $p\left(W_{1}\right) \cap p(T \times[0,1])=\partial p\left(W_{1}\right)=\partial(T \times[0,1])=$ $T$. Now $p\left(W_{1}\right)$ is a copy of $\mathrm{W}$, and by Corollary $(3.12) p(T \times[0,1])$ is a copy of $W$ also.

Suppose both $T_{1}$ and $T_{2}$ are nonseparating. We obtain the decomposition $\tilde{M}=\left(T_{1}\right.$ $\times[0,1]) \cup\left(T_{2} \times[0,1]\right)$. Then $M=p\left(T_{1} \times[0,1]\right) \cup p\left(T_{2} \times[0,1]\right)$ and necessarily $p\left(T_{1} \times[0,1]\right) \cap p\left(T_{2} \times[0,1]\right)=T$. By Corollary $(3.12), p\left(T_{i} \times[0,1]\right)$ is a copy of $W, i=1,2$.

(2) Suppose $p^{-1}(T)=\tilde{T}$ is a single torus. By Lemma (5.3), $\tilde{T}$ decomposes $\tilde{M}$ into 2 copies of $W: \tilde{M}=\tilde{W}_{1} \cup \tilde{W}_{2}$ with $\tilde{W}_{1} \cap \tilde{W}_{2}=\partial \tilde{W}_{1}=\partial \tilde{W}_{2}=\tilde{T}$. Then $M=p\left(\tilde{W}_{1}\right) \cup$ $p\left(\tilde{W}_{2}\right)$ and necessarily $p\left(\tilde{W}_{1}\right) \cap p\left(\tilde{W}_{2}\right)=T$. By Corollary (4.9), $p\left(\tilde{W}_{i}\right)$ is a copy of $W, i=1,2$. Q.E.D.

Main Lemma (6.3). Suppose $M$ is not a torus bundle over $S^{1}$. If $\iota: M \rightarrow M$ is an involution with fixed points, then there is a separating incompressible torus $T \subset M$ with $\iota(T)=T$ and with no fixed points of $\iota$ on $T$.

Proof. Note by Proposition (6.1) each incompressible torus in $M$ is separating. By Theorem (2.7) there is an incompressible torus $T \subset M$ with either $T \cap \iota(T)=\varnothing$ or with $\iota(T)=T$ and with no fixed points of $\iota$ on $T$.

Suppose $T \cap \iota(T)=\varnothing$. By Lemmas (6.2) and (5.2) we have the following decomposition of $M: M=W_{1} \cup T \times[0,1] \cup W_{2}$, where $W_{1}, W_{2}$ are disjoint copies of $W$ with $W_{1} \cap T \times[0,1]=\partial W_{1}=T \times 0=T$ and $W_{2} \cap T \times[0,1]=\partial W_{2}=T \times$ $1=\iota(T)$.

Necessarily, $\iota\left(W_{1}\right)=W_{2}$ and $\iota(T \times[0,1])=T \times[0,1]$. By the same arguments as in part (2) of the proof of Main Lemma (5.4) a nonseparating incompressible torus $T \subset M$ can be constructed. Thus $T \cap \iota(T)=\varnothing$ is not possible. Q.E.D.

TheOREM (6.4). Suppose $M$ is not a torus bundle over $S^{1}$ and $\iota: M \rightarrow M$ is an involution with fixed points. Then ı has exactly 2 fixed points.

Proof. Let $T \subset M$ be the torus of Main Lemma (6.3). By Lemma (6.2), $T$ decomposes $M$ into 2 copies $W_{1}, W_{2}$ of $W$ :

$$
M=W_{1} \cup W_{2} \text { and } W_{1} \cap W_{2}=\partial W_{1}=\partial W_{2}=T
$$

We must have $\iota\left(W_{i}\right)=W_{i}, i=1,2$. By Corollary (4.8), $\iota$ has either 0 or 2 fixed points in $W_{i}, i=1,2$. Hence $\iota$ has either 2 or 4 fixed points.

Suppose $\iota$ has 4 fixed points. Then $\left.\iota\right|_{W_{i}}$ has 2 fixed points, $i=1,2$. Consider $D(W)=W \cup W^{\prime}$ and the involution $D\left(\rho_{2}\right): D(W) \rightarrow D(W)$. By Theorem (4.7), there is an isomorphism $h_{1}: W \rightarrow W_{1}$ so that $\left.\iota\right|_{W_{1}}=h_{1} \rho_{2} h_{1}^{-1}$. Then $\left.\iota\right|_{\partial W_{2}}=$ $h_{1} \rho_{2}\left(\left.h_{1}\right|_{\partial W_{2}}\right)^{-1}$. Again by Theorem (4.7) there is an isomorphism $h_{2}: W^{\prime} \rightarrow W_{2}$ with $\left.\iota\right|_{W_{2}}=h_{2} \rho_{2} h_{2}^{-1}$ extending $\left.h_{1}\right|_{\partial W_{2}}$. Thus there is an isomorphism $h=h_{1} \cup h_{2}: D(W)$ $\rightarrow M$. Therefore $M=D(W)=M_{2}$ and $M$ is a torus bundle over $S^{1}$, a contradiction. Q.E.D. 
THEOREM (6.5). Let $M, M^{\prime}$ each be unions along the boundary of 2 orientable twisted I-bundles over Klein bottles and let $\iota: M \rightarrow M, \iota^{\prime}: M^{\prime} \rightarrow M^{\prime}$ be involutions with fixed points. If $\iota, \iota$ have the same number of fixed points, then there exists an isomorphism $h$ : $M \rightarrow M^{\prime}$ satisfying $\iota^{\prime}=h \iota h^{-1}$.

Proof. By Theorem (6.4) and Corollary (5.7) we may assume that $M, M^{\prime}$ are not torus bundles over $S^{1}$ and that $\iota, \iota^{\prime}$ have 2 fixed points each. As in the proof of Theorem (6.4) we have decompositions of $M, M^{\prime}$ into copies of $W$ with regard to $\iota$, $\iota^{\prime}$, respectively: $M=W_{1} \cup W_{2}$ and $M^{\prime}=W_{1}^{\prime} \cup W_{2}^{\prime}$. We may assume that $\iota W_{W_{1}}$, $\left.\iota^{\prime}\right|_{W_{1}^{\prime}}$ are fixed point free and that $\left.\iota\right|_{W_{2}},\left.\iota^{\prime}\right|_{W_{2}^{\prime}}$ have 2 fixed points each. By Theorem (4.7) there is an isomorphism $h_{1}: W_{1} \rightarrow W_{1}^{\prime}$ with $\left.\iota^{\prime}\right|_{W_{1}^{\prime}}=h_{1} \iota h_{1}^{-1}$ and there is an isomorphism $h_{2}: W_{2} \rightarrow W_{2}^{\prime}$ with $\left.\iota^{\prime}\right|_{W_{2}^{\prime}}=h_{2} \iota h_{2}^{-1}$ and extending $\left.h_{1}\right|_{\partial W_{2}}$. Then $h=h_{1}$ $\cup h_{2}: M \rightarrow M^{\prime}$ is an isomorphism with $\iota^{\prime}=h \iota h^{-1}$. Q.E.D.

COROLlaRY (6.6). The only unions along the boundary of 2 orientable twisted I-bundles over Klein bottles that admit involutions with fixed points are $M_{2}, M_{6}$. Moreover, all such involutions on $M_{2}, M_{6}$ are conjugate to $\iota_{4}, \iota_{2}$, respectively.

Proof. $M_{1}=S^{1} \times S^{1} \times S^{1}$ is not the union along the boundary of 2 orientable twisted $I$-bundles over Klein bottles. (There is no epimorphism $H_{1}(W) \oplus H_{1}(W) \rightarrow$ $H_{1}\left(S^{1} \times S^{1} \times S^{1}\right)$.

Corollary (5.7), Theorems (6.4) and (6.5) complete the proof. Q.E.D.

Finally, Corollaries (5.7) and (6.6) prove our main result.

THEOREM (6.7). The only space forms from the orientable ones $M_{1}, \ldots, M_{6}$ which admit involutions with fixed points are $M_{1}, M_{2}, M_{6}$. Moreover these involutions are unique up to conjugacy and have 8, 4, 2 fixed points respectively.

7. The orbit manifolds $M_{1}^{*}, M_{2}^{*}$, and $M_{6}^{*}$. In this section we exhibit canonical decompositions of the manifolds $M_{1}^{*}, M_{2}^{*}$, and $M_{6}^{*} . P^{2}$ denotes the projective plane.

Proposition (7.1). Let $C$ be a 3-cell and let $\iota: C \rightarrow C$ be an involution with fixed point $x_{0}$. Suppose $C_{0} \subset$ int $C$ is a 3-cell with $x_{0} \in$ int $C_{0}$ and $\iota\left(C_{0}\right)=C_{0}$. Then $\overline{C-C_{0}} / \iota=P^{2} \times[0,1]$.

Proof. We may assume that $\overline{C-C_{0}}=S^{2} \times[0,1]$ and by Lemma (3.1) that $\iota(x, t)=(-x, t)$. Q.E.D.

Definition. A projective solid $V^{*}$ is defined as follows. Let $P_{1}, P_{2}$ be projective planes, let $D_{i} \subset P_{i} \times 1$ be 2-cells, $i=1,2$, and let $h: D_{1} \rightarrow D_{2}$ be an isomorphism. Define

$$
V^{*}=P_{1} \times[0,1] \cup P_{2} \times[0,1] /(x, 1) \sim(h(x), 1) \text { for } x \in D_{1} .
$$

The usual notation is $V^{*}=\left(P_{1} \times[0,1]\right) \#_{\partial}\left(P_{2} \times[0,1]\right) . V^{*}$ does not depend on the choice of $D_{1}, D_{2}$, or $h$. Note that $\partial V^{*}$ consists of 2 projective planes, $P_{1} \times 0$, $P_{2} \times 0$, and a Klein bottle, $\left(P_{1} \times 1\right) \#\left(P_{2} \times 1\right)$.

Proposition (7.2). Let $V$ be a solid torus and let $\iota: V \rightarrow V$ be an involution with fixed points $x_{1}, x_{2}$. Suppose $C_{1}, C_{2} \subset$ int $V$ are disjoint 3-cells with $x_{i} \in \operatorname{int} C_{i}$ and $\iota\left(C_{i}\right)=C_{i}, i=1,2$. Then $\overline{V-\left(C_{1} \cup C_{2}\right)} / \iota \mid=V^{*}$. 
Proof. By Corollary (3.4) we may assume that $\iota=\iota_{2}$. For $\iota_{2}$ the proposition is easily verified. Q.E.D.

Definition. Let $V_{1}^{*}, V_{2}^{*}$ be 2 projective solids. On the Klein bottles $K_{i} \subset \partial V_{i}^{*}$ we choose nonseparating annuli $A_{i}, i=1,2$. By Lemma (4.1), $A_{i}$ is uniquely determined up to an ambient isotopy on $K_{i}$ and hence on $V_{i}^{*}, i=1,2$. Let $h: A_{1} \rightarrow A_{2}$ be an isomorphism. Define

$$
T \times[0,1]^{*}=V_{1}^{*} \cup V_{2}^{*} / x \sim h(x) \text { for } x \in A_{1} .
$$

$T \times[0,1]^{*}$ does not depend on the choice of $h$.

Note that $\partial\left(T \times[0,1]^{*}\right)$ consists of 4 projective planes and 1 torus.

Proposition (7.3). Let $\iota: T \times[0,1] \rightarrow T \times[0,1]$ be an involution with fixed points $x_{1}, x_{2}, x_{3}, x_{4}$. Suppose $C_{1}, C_{2}, C_{3}, C_{4} \subset \operatorname{int}(T \times[0,1])$ are disjoint 3-cells with $x_{i} \in$ int $C_{i}$ and with $\iota\left(C_{i}\right)=C_{i}, i=1,2,3,4$. Then

$$
\overline{T \times[0,1]-\left(C_{1} \cup C_{2} \cup C_{3} \cup C_{4}\right)} / \iota=T \times[0,1]^{*} .
$$

Proof. By Corollary (3.11) we may assume that $\iota=\kappa \times \kappa \times \tau$. The proposition is easily verified in this case. Q.E.D.

LeMma (7.4). Let $K$ be a Klein bottle and let $S_{1}, S_{2} \subset K$ be nontrivial separating 1 -spheres. Then there is an ambient isotopy on $K$ that maps $S_{1}$ onto $S_{2}$.

Proof. This is well known. We sketch a brief proof. Let $K=S^{1} \times[0,1] /(x, 0) \sim$ $(\bar{x}, 1)$ and let $q: S^{1} \times[0,1] \rightarrow K$ be the natural identification map. Then $\pi_{1}(K)$ $=\left\langle a, b: b a b^{-1}=a^{-1}\right\rangle$ where $a=\left[q\left(S^{1} \times 0\right)\right]$ and $b=[q(1 \times[0,1])]$. Now $S_{i}$ is the boundary of a Moebius strip which is the regular neighborhood of a 1-sphere $S_{i}^{\prime}$. We can find a 2-sided 1-sphere in $K$ which intersects $S_{i}^{\prime}$ transversally in a single point. Thus we can apply, [3, Lemma 2.13, p. 23], and conclude that $\left[S_{i}^{\prime}\right]=a^{r_{i}} b^{ \pm 1}, i=1,2$. But $\left[S_{i}\right]=a^{r_{i}} b^{ \pm 1} a^{r_{i}} b^{ \pm 1}, i=1,2$. It follows from the identity on p. 23 of [3] that $\left[S_{i}\right]=b^{ \pm 2}, i=1,2$. Hence there are embeddings $f_{i}: S^{1} \rightarrow K$ with $f_{i}\left(S^{1}\right)=S_{i}$, $i=1,2$, and with $f_{1}$ and $f_{2}$ homotopic. Then there is an ambient isotopy that maps $S_{1}$ onto $S_{2}$ (see e.g. [3, Lemma 2.10, p. 21]). Q.E.D.

Definition. Let $V^{*}$ be a projective solid and let $N$ be a solid Klein bottle. Let $K \subset \partial V^{*}$ be the Klein bottle. On $K$ we choose a nonseparating annulus $A_{1}$ and on $\partial N$ a separating annulus $A_{2}$ that does not bound a 2-cell on $\partial N$. By Lemmas (4.1) and (7.4), $A_{1}, A_{2}$ are uniquely determined up to ambient isotopy on $K, \partial N$ respectively. Let $h: A_{1} \rightarrow A_{2}$ be an isomorphism. Define

$$
W^{*}=V^{*} \cup N / x \sim h(x) \text { for } x \in A_{1} \text {. }
$$

$W^{*}$ does not depend on the choice of $h$.

Note that $\partial W^{*}$ consists of 2 projective planes and 1 Klein bottle.

Proposition (7.5). Let $\iota: W \rightarrow W$ be an involution with fixed points $x_{1}, x_{2}$. Suppose $C_{1}, C_{2} \subset$ int $W$ are disjoint 3-cells with $x_{i} \in$ int $C_{i}$ and with $\iota\left(C_{i}\right)=C_{i}, i=1,2$. Then $\overline{W-C_{1} \cup C_{2}} / \iota \mid=W^{*}$.

Proof. By Corollary (4.8) we may assume that $\iota=\rho_{2}$. It is not difficult to verify the proposition in this case. Q.E.D. 
Definition. Let $\left(T_{1} \times[0,1]\right)^{*},\left(T_{2} \times[0,1]\right)^{*}$ be 2 copies of $(T \times[0,1])^{*}$, let $T_{i}^{\prime} \subset \partial\left(T_{i} \times[0,1]\right)^{*}$ be the torus, $i=1,2$, and let $h: T_{1}^{\prime} \rightarrow T_{2}^{\prime}$ be an arbitrary isomorphism. Define

$$
M_{1}^{*}=\left(T_{1} \times[0,1]\right)^{*} \cup\left(T_{2} \times[0,1]\right)^{*} / x \sim h(x) \text { for } x \in T_{1}^{\prime} .
$$

Note that $\partial M_{1}^{*}$ consists of 8 projective planes.

Let $\iota: T \times[0,1] \rightarrow T \times[0,1]$ be an orientation reversing fixed point free involution with $\iota(T \times 0)=T \times 1$. By Corollary (3.12), then $T \times[0,1] / \iota=M O \times S^{1}, M O$ a Moebius strip.

Definition. Let $T^{\prime} \subset \partial(T \times[0,1])^{*}$ be the torus and let $h: T^{\prime} \rightarrow \partial\left(M O \times S^{1}\right)$ be an arbitrary isomorphism. Define

$$
M_{2}^{*}=(T \times[0,1])^{*} \cup M O \times S^{1} / x \sim h(x) \text { for } x \in T^{\prime} .
$$

Note that $\partial M_{2}^{*}$ consists of 4 projective planes.

Let $\iota: W \rightarrow W$ be an orientation reversing fixed point free involution. By Corollary (4.9), then $W / \iota=W / \rho_{0}$ is the nonorientable twisted $I$-bundle over a Klein bottle.

Definition. Let $K \subset \partial W^{*}$ be the Klein bottle and let $h: K \rightarrow \partial\left(W / \rho_{0}\right)$ be an arbitrary isomorphism. Define

$$
M_{6}^{*}=W^{*} \cup W / \rho_{0} / x \sim h(x) \text { for } x \in K .
$$

Note that $\partial M_{6}^{*}$ consists of 2 projective planes.

It will follow from Theorem (7.6) that $M_{1}^{*}, M_{2}^{*}, M_{6}^{*}$ do not depend on the choice of $h$.

TheOREM (7.6). Let $\iota: M_{i} \rightarrow M_{i}, i=1,2,6$ be an involution with isolated fixed points $x_{1}, \ldots, x_{k}, k=8,4,2$ respectively. Suppose $C_{1}, \ldots, C_{k} \subset M_{i}$ are disjoint 3-cells with $x_{j} \in$ int $C_{j}$ and $\iota\left(C_{j}\right)=C_{j}, j=1, \ldots, k$. Then

$$
\overline{M_{i}-\left(C_{1} \cup \cdots \cup C_{k}\right)} / \iota=M_{i}^{*}, \quad i=1,2,6 .
$$

Also $M_{2}^{*}=D\left(W^{*}\right)$, the double of $W^{*}$.

Proof. Let $p: \tilde{M}_{i} \rightarrow M_{i}^{*}$ be the orientable double covering. Adding $k$ 3-cells to $\partial \tilde{M}_{i}$, we obtain the closed manifold $\hat{M}_{i}$. The deck transformation on $\tilde{M}_{i}$ extends to an involution on $\hat{M}_{i}$ with $k$ fixed points.

If $i=1, p^{-1}\left(T_{j} \times[-1,1]\right)^{*}, j=1,2$, are 2 copies of $T \times[0,1]$ with four 3 -cells removed from each.

If $i=2, p^{-1}(T \times[-1,1])^{*}$ is a copy of $T \times[0,1]$ with four 3-cells removed, and $p^{-1}\left(M O \times S^{1}\right)$ is another copy of $T \times[-1,1]$.

If $i=6, p^{-1}\left(W^{*}\right)$ is a copy of $W$ with two 3-cells removed, and $p^{-1}\left(W / \rho_{2}\right)$ is another copy of $W$.

Therefore $\hat{M}_{1}, \hat{M}_{2}$ are torus bundles over $S^{1}$ and $\hat{M}_{6}$ is the union along the boundary of 2 orientable twisted $I$-bundles over Klein bundles. By Corollaries (5.7) and (6.6), $\hat{M}_{i}=M_{i}, i=1,2,6$, and by uniqueness of involutions we obtain an isomorphism $h: \tilde{M}_{i} \rightarrow \overline{M_{i}-\left(C_{1} \cup \cdots \cup C_{k}\right)}$ which commutes with the deck transformation on $\tilde{M}_{i}$ and with $\left.\iota\right|_{\overline{M_{i}-\left(C_{1} \cup \cdots \cup C_{k}\right)}}$. Therefore $h$ defines an isomorphism $\bar{h}$ : $M_{i}^{*} \rightarrow \overline{M-\left(C_{1} \cup \cdots \cup C_{k}\right)} / \iota \mid$ 
Considering the orientable double covering of $D\left(W^{*}\right)$ we obtain similarly $\hat{M}$, and we conclude by Corollary (6.6) that $\hat{M}=M_{2}$, and then that $M_{2}^{*}=D\left(W^{*}\right)$. Q.E.D.

THEOREM (7.7). There are natural double coverings $M_{1}^{*} \stackrel{2}{\rightarrow} M_{2}^{*} \stackrel{2}{\rightarrow} M_{6}^{*}$.

Proof. (1) We may assume that $M_{1}^{*}=D(T \times[0,1])^{*}$, the double of $(T \times[0,1])^{*}$. We decompose $M_{1}^{*}$ by a bicollar $T^{\prime} \times[-1,1] \subset M_{1}^{*}$ of the torus $T^{\prime} \subset \partial(T \times[0,1])^{*}$ :

$$
M_{1}^{*}=\left(T_{-} \times[0,1]\right)^{*} \cup T^{\prime} \times[-1,1] \cup\left(T_{+} \times[0,1]\right)^{*}
$$

with

$$
\left(T_{i} \times[0,1]\right)^{*} \cap T^{\prime} \times[-1,1]=T^{\prime} \times i 1, \quad i+,-.
$$

Define the involution $\lambda: M_{1}^{*} \rightarrow M_{1}^{*}$ as follows. Let $\left.\lambda\right|_{T^{\prime} \times[-1,1]}=\alpha \times$ id $\times \tau$ : $T^{\prime} \times[-1,1] \rightarrow T^{\prime} \times[-1,1]$ (see Corollary (3.11)) and extend the isomorphism $\left.\lambda\right|_{T^{\prime} \times-1}: T^{\prime} \times-1 \rightarrow T^{\prime} \times 1$ to an isomorphism

$$
\left.\lambda\right|_{\left(T_{-} \times[0,1]\right)^{*}}:\left(T_{-} \times[0,1]\right)^{*} \rightarrow\left(T_{+} \times[0,1]\right)^{*} .
$$

Let

$$
\lambda=\left.\left.\lambda\right|_{\left(T_{-} \times[0,1]\right)^{*}} \cup \lambda\right|_{T^{\prime} \times[-1,1]} \cup\left(\left.\lambda\right|_{\left(T_{-} \times[0,1]\right)^{*}}\right)^{-1} .
$$

Then $\lambda$ is fixed point free and

$$
M_{1}^{*} / \lambda=\left(T_{-} \times[0,1]\right)^{*} \cup T^{\prime} \times[-1,1] / \lambda \mid=M_{2}^{*} .
$$

(2) We may assume that $M_{2}^{*}=D\left(W^{*}\right)$, the double of $W^{*}$. We decompose $M_{2}^{*}$ by a bicollar $K \times[-1,1] \subset M_{2}^{*}$ of the Klein bottle $K \subset \partial W^{*}$ :

$$
M_{2}^{*}=W_{+}^{*} \cup K \times[-1,1] \cup W_{-}^{*} \quad \text { with } W_{i}^{*} \cap K \times[-1,1]=K \times i 1, \quad i=+,- \text {. }
$$

Define the involution $\mu: M_{2}^{*} \rightarrow M_{2}^{*}$ as follows. Let $\left.\mu\right|_{K \times[-1,1]}: K \times[-1,1] \rightarrow K \times$ $[-1,1]$ be the involution with $K \times[-1,1] / \mu \mid=$ nonorientable twisted $I$-bundle over the Klein bottle. Extend the isomorphism $\left.\mu\right|_{K \times-1}: K \times-1 \rightarrow K \times 1$ to an isomorphism $\left.\mu\right|_{W_{-}^{*}}: W_{-}^{*} \rightarrow W_{+}^{*}$. Let

$$
\mu=\left.\left.\mu\right|_{W_{-}^{*}} \cup \mu\right|_{K \times[-1,1]} \cup\left(\left.\mu\right|_{W_{-}^{*}}\right)^{-1} .
$$

Then $\mu$ is fixed point free and

$$
M_{2}^{*} / \mu=W_{-}^{*} \cup K \times[-1,1] / \mu \mid=M_{6}^{*} \text {. Q.E.D. }
$$

\section{REFERENCES}

1. D. B. A. Epstein, Projective planes in 3-manifolds, Proc. London Math. Soc. (3) 11 (1961), 469-484.

2. W. Hantzsche and H. Wendt, Dreidimensionale euklidische Raumformen, Math. Ann. 110 (1935), $593-611$.

3. J. Hempel, 3-manifolds, Ann. of Math. Studies, no. 86, Princeton Univ. Press, Princeton, N.J., 1976.

4. K. Kwun and J. Tollefson, PL involutions of $S^{1} \times S^{1} \times S^{1}$, Trans. Amer. Math. Soc. 203 (1975), 97-106.

5. G. R. Livesay, Involutions with two fixed points on the three-sphere, Ann. of Math. (2) 78 (1963), $582-593$.

6. E. Luft, Equivariant surgery on essential annuli and incompressible tori with respect to involutions (to appear). 
7. E. Luft and D. Sjerve, 3-manifolds with subgroups $\mathbf{Z} \oplus \mathbf{Z} \oplus \mathbf{Z}$ in their fundamental groups, Pacific J. Math. (to appear).

8. P. Orlik, Seifert manifolds, Lecture Notes in Math., vol. 291, Springer-Verlag, Berlin and New York, 1972.

9. C. Rourke and B. Sanderson, Introduction to piecewise-linear topology, Ergeb. Math. Grenzgeb., no. 69, Springer, Berlin and New York, 1972.

10. J. Tollefson, Involutions of sufficiently large 3-manifolds, Topology 20 (1981), 323-352.

11. J. Wolf, Spaces of constant curvature, McGraw-Hill, New York, 1967.

Department of Mathematics, The University of British Columbia, Vancouver,.British Co LUmbia, Canada V6T 1W5 